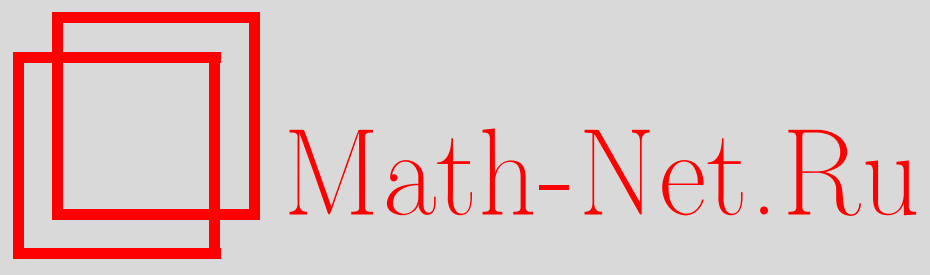

J. Dedecker, F. Merlevede, Convergence rates in the law of large numbers for Banach-valued dependent variables, Теория вероятн. и ее примен., 2007, том 52, выпуск $3,562-587$

DOI: https://doi.org/10.4213/tvp78

Использование Общероссийского математического портала Math-Net.Ru подразумевает, что вы прочитали и согласны с пользовательским соглашением

http://www . mathnet.ru/rus/agreement

Параметры загрузки:

IP : 34.227 .88 .159

26 апреля 2023 г., 18:27:19

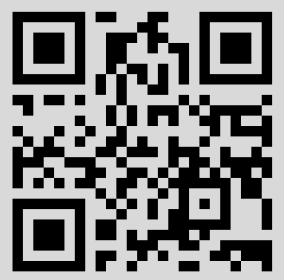




\section{CONVERGENCE RATES IN THE LAW OF LARGE NUMBERS FOR BANACH-VALUED DEPENDENT VARIABLES ${ }^{1)}$}

Усиленный закон больших чисел Марцинкевича-Зигмунда для мартингалов мы распространяем на слабозависимые случайные величины со значениями в гладких банаховых пространствах. Условия выражены в терминах условных математических ожиданий. В случае гильбертовых пространств мы показываем, что наши условия слабее, чем оптимальные для последовательностей со свойством сильного перемешивания (которые ранее были известны только для действительнозначных величин). В качестве следствия получены скорости сходимости для статистики Крамера-Мизеса и для эмпирической оценки ковариационного оператора гильбертовозначного процесса авторегрессии.

Ключевые слова и фразы: гладкие банаховы пространства, гильбертовы пространства, усиленный закон больших чисел Марцинкевича-Зигмунда, сходимость почти наверное, мартингалы, слабая зависимость, статистика Крамера-Мизеса.

\section{Introduction}

The problem of rates of convergence in the strong law of large numbers for i.i.d. random variables with moments of order $1 \leqslant p<2$ has been solved by Baum and Katz [3] for real-valued variables, and by [1] for variables with values in Banach spaces of type $p$. To our knowledge the first extensions of this type of results to martingale differences sequences were derived by Woyczyński [29] who considered random variables with values in smooth separable Banach spaces (see Definition 2 and Theorem 1, Section 2). Starting from Woyczyński's result and using a coboundary decomposition due to Gordin [11], Theorem 1 can be extended to stationary sequences under mixingale-type conditions (see Theorem 3 , Subsection 2.2). At the boundary (the case $p=2$ ) this criterion is the same as that given by Gordin [11] for the central limit theorem.

* Université Paris VI, Laboratoire de Statistique Théorique et Appliquée, Boîte 158, Plateau A, 175, rue du Chevaleret, 75013 Paris, France; e-mail: dedecker@ccr.jussieu.fr

** Laboratoire de Probabilités et Modèles Aléatoires, Université Paris VI, C.N.R.S. UMR 7599, Boîte 188, 175, rue du Chevaleret, 75013 Paris, France; e-mail: merleve@ccr.jussieu.fr. 
For strongly mixing real-valued random variables, this approach does not lead to optimal results, as quoted in Section 3. Concerning the convergence rates in the strong law of large numbers for weakly dependent sequences of real-valued random variables, we mention the papers by Lai [14], Hipp [13], Berbee [4], Peligrad [19], [20], Shao [26] and Rio [22]. In the two latter ones, the optimality of the results is discussed.

In Theorem 4 of Section 3, we establish convergence rates in the strong law for partial sums of Hilbert-valued random variables under a projective criterion, which seems to be new even in the real case. This criterion can be viewed as a mixingale-type condition, since it is verified by martingale differences sequences and leads to the optimal results for strongly mixing processes. At the boundary (the case $p=2$ ) our condition is the same as that obtained in $[8$, Corollary $2(\beta)]$ for the central limit theorem in Hilbert spaces. This generalization is important, since it covers a much broader line of examples than mixing processes (see the examples in [16] and [7]). The key of the proof of Theorem 4 is a new maximal inequality (see Proposition 1, Section 3) in which the dependence coefficients involved are expressed in terms of conditional expectations. This maximal inequality is more precise than a related one stated in [17, Lemma 3.3]. As in [17], the proof combines a martingale approximation of blocks as done in [26] and the quantile method as developed in [22], [23].

In Proposition 2 of Section 4, we apply Theorem 4 to describe the asymptotic behavior of Cramér-von Mises statistics obtained from dependent variables. In this context, it is natural to consider the empirical distribution function as a random variable with values in $\mathbf{L}^{2}(\mu)$ for an appropriate measure $\mu$ on the real line. As a by-product, we obtain the asymptotic behavior of the supremum of the empirical process over generalized Sobolev balls (the class $W_{1}(\mu)$ defined in Lemma 1 ).

In Section 5, we study the empirical covariance operator $C_{n}$ of a stationary Hilbert-valued autoregressive process. Such processes may be useful when considering estimation and forecasting problems for several classes of continuous time processes (see [5] for more details). In Proposition 3 we give the rates of convergence of $C_{n}$ to the covariance operator $C_{X}$ when the innovations have moments of order $2<p \leqslant 4$.

\section{The case of smooth separable Banach spaces}

In order to develop our result, we need some definitions.

D e f i n i t i o n 1 . Let $(\Omega, \mathscr{A}, \mathbf{P})$ be a probability space and $\left(\mathbf{B},\|\cdot\|_{\mathbf{B}}\right)$ a separable Banach space. For any real $p \geqslant 1$, denote by $\mathbf{L}_{\mathbf{B}}^{p}$ the space of $\mathbf{B}$ valued random variables such that $\|X\|_{\mathbf{L}_{\mathbf{B}}^{p}}^{p}=\mathbf{E}\|X\|_{\mathbf{B}}^{p}$ is finite. Let $\left(\mathscr{M}_{i}\right)_{i>0}$ be an increasing sequence of sub- $\sigma$-algebras of $\mathscr{A}$. We say that $\left(X_{i}\right)_{i>0}$ is 
a sequence of $\mathbf{B}$-valued martingale differences (with respect to the filtration $\left.\left(\mathscr{M}_{i}\right)_{i>0}\right)$ if:

1) For any positive $i, X_{i}$ is $\mathscr{M}_{i}$-measurable and belongs to $\mathbf{L}_{\mathbf{B}}^{1}$;

2) for any $i>1, \mathbf{E}\left(X_{i} \mid \mathscr{M}_{i-1}\right)=0$ almost surely.

Definition 2. Following [21], we say that a Banach space $\left(\mathbf{B},\|\cdot\|_{\mathbf{B}}\right)$ is $r$-smooth $(1<r \leqslant 2)$ if there exists an equivalent norm $\|\cdot\|$ such that

$$
\sup _{t>0}\left\{\frac{1}{t^{r}} \sup \{\|x+t y\|+\|x-t y\|-2:\|x\|=\|y\|=1\}\right\}<\infty .
$$

Clearly, if $\mathbf{B}$ is $r$-smooth, then it is $r^{\prime}$-smooth for any $r^{\prime} \leqslant r$. A Banach space is said to be superreflexive if it is $r$-smooth for some $1<r \leqslant 2$. From [2] we know that if $\mathbf{B}$ is $r$-smooth and separable, then there exists a constant $D$ such that, for any sequence of $\mathbf{B}$-valued martingale differences $\left(X_{i}\right)_{i \geqslant 1}$,

$$
\mathbf{E}\left\|X_{1}+\cdots+X_{n}\right\|_{\mathbf{B}}^{r} \leqslant D \sum_{i=1}^{n} \mathbf{E}\left\|X_{i}\right\|_{\mathbf{B}}^{r}
$$

From (2.1) we see that $r$-smooth Banach spaces play the same role for martingales as spaces of type $r$ do for sums of independent variables. When the constant $D$ needs to be specified, we shall say that $\mathbf{B}$ is $(r, D)$-smooth. Note that, for any measurable space $(T, \mathscr{A}, \nu), \mathbf{L}^{p}(T, \mathscr{A}, \nu)$ is $(p \wedge 2)$-smooth for any $p>1$, and that any separable Hilbert space is $(2,1)$-smooth.

$\mathrm{D}$ e f i n i t i o n 3. For any nonincreasing càdlàg function $f$ from $\mathbf{R}^{+}$ to $\mathbf{R}^{+}$, define the generalized inverse $f^{-1}(u)=\inf \{t \geqslant 0: f(t) \leqslant u\}$. For any nonnegative random variable $X$, define the upper tail function $L_{X}(t)=$ $\mathbf{P}(X>t)$ and the quantile function $Q_{X}=L_{X}^{-1}$. Let $\left(X_{i}\right)_{i>0}$ be a sequence of B-valued random variables. Following [29], we write $\left(X_{i}\right) \prec X$ if there exists a positive random variable $X$ such that $Q_{X} \geqslant \sup _{k \geqslant 1} Q_{\left\|X_{k}\right\|_{\mathbf{B}}}$.

2.1. The martingale case. In [29] the following theorem is proved.

Theorem 1. Let $\mathbf{B}$ be a separable Banach space and $\left(X_{i}\right)_{i>0}$ a sequence of $\mathbf{B}$-valued martingale differences. Assume that $\left(X_{i}\right) \prec X$ for some positive random variable $X$ and define $S_{n}=X_{1}+\cdots+X_{n}$.

1. Let $1<p<2$. If $X$ belongs to $\mathbf{L}_{\mathbf{R}}^{p}$ and $\mathbf{B}$ is $r$-smooth for some $r>p$, then $n^{-1 / p} S_{n}$ tends to 0 almost surely.

2. If $\mathbf{E}\left(X \ln ^{+} X\right)<\infty$ and $\mathbf{B}$ is superreflexive, then $n^{-1} S_{n}$ tends to 0 almost surely.

The next theorem is slightly more precise than the result of [29], as we shall see in Remarks 1 and 2. The proof of Theorem 2 is given in the appendix.

Theorem 2. Let $\mathbf{B},\left(X_{i}\right)_{i>0}, X$, and $S_{n}$ be defined as in Theorem 1 . 
1. Let $1<p<2$. If $X$ belongs to $\mathbf{L}_{\mathbf{R}}^{p}$ and $\mathbf{B}$ is $r$-smooth for some $r>p$, then, for any $1 \leqslant 1 / \alpha \leqslant p$ and all $\varepsilon>0$,

$$
\sum_{n=1}^{\infty} n^{\alpha p-2} \mathbf{P}\left(\max _{1 \leqslant k \leqslant n}\left\|S_{k}\right\|_{\mathbf{B}} \geqslant \varepsilon n^{\alpha}\right)<\infty \quad \text { for each } \varepsilon>0 .
$$

2. If $\mathbf{E}\left(X \ln ^{+} X\right)<\infty$ and $\mathbf{B}$ is superreflexive, then for all $\varepsilon>0$,

$$
\sum_{n=1}^{\infty} \frac{1}{n} \mathbf{P}\left(\max _{1 \leqslant k \leqslant n}\left\|S_{k}\right\|_{\mathbf{B}} \geqslant \varepsilon n\right)<\infty \quad \text { for each } \varepsilon>0 .
$$

$\mathrm{R}$ e $\mathrm{m}$ a $\mathrm{rk} 1$. The sequence $\max _{1 \leqslant k \leqslant n}\left\|S_{k}\right\|_{\mathrm{B}}$ being nondecreasing, property (2.2) with $\alpha p=1$ is equivalent to

$$
\sum_{N=1}^{\infty} \mathbf{P}\left(\max _{1 \leqslant k \leqslant 2^{N}}\left\|S_{k}\right\|_{\mathbf{B}} \geqslant \varepsilon 2^{N / p}\right)<\infty .
$$

We infer from (2.4) that $n^{-1 / p} S_{n}$ tends to 0 almost surely, so that Theorem 2 contains Theorem 1.

$\mathrm{R}$ e $\mathrm{m}$ a r k 2. Property (2.2) describes rate of convergence in the strong law. Indeed, by [14, Lemma 4], it implies in case $\alpha p>1$ that

$$
\sum_{n=1}^{\infty} n^{\alpha p-2} \mathbf{P}\left(\sup _{k \geqslant n} k^{-\alpha}\left\|S_{k}\right\|_{\mathbf{B}} \geqslant \varepsilon\right)<\infty .
$$

Since the probabilities in (2.5) are nonincreasing in $n$, it follows that

$$
\mathbf{P}\left(\sup _{k \geqslant n} k^{-\alpha}\left\|S_{k}\right\|_{\mathbf{B}} \geqslant \varepsilon\right)=o\left(\frac{1}{n^{\alpha p-1}}\right) .
$$

$\mathrm{R}$ e $\mathrm{m}$ a r k 3 . The moment condition $\mathbf{E}\left(X \ln ^{+} X\right)<\infty$ in item 2 of Theorems 1 and 2 cannot be removed. More precisely, in [10] the following result is proved: if $Y$ is any real-valued integrable and centered random variable such that $\mathbf{E}\left(|Y| \ln ^{+}|Y|\right)=\infty$, then there exists a sequence $\left(X_{i}\right)_{i>0}$ of martingale differences with the same marginal distribution as $Y$ and such that $n^{-1} S_{n}$ diverges almost surely.

$\mathrm{R}$ e $\mathrm{m}$ a $\mathrm{r} \mathrm{k}$ 4. To be complete about strong laws of large numbers, let us quote the following remarkable result, which is a particular case of in [28, Theorem 1]. For a separable Banach space B, the following two conditions are equivalent:

1) $\mathbf{B}$ is $r$-smooth;

2) for every sequence $\left(X_{n}\right)_{n>0}$ of martingale differences with values in $\mathbf{B}$ such that $\sum n^{-r} \mathbf{E}\left\|X_{n}\right\|_{\mathbf{B}}^{r}<\infty$, we have that $n^{-1} S_{n}$ tends to 0 almost surely and in $\mathbf{L}_{\mathbf{B}}^{r}$. 
2.2. Application to stationary sequences. Let $(\Omega, \mathscr{A}, \mathbf{P})$ be a probability space and $T: \Omega \mapsto \Omega$ a bijective bimeasurable transformation preserving the probability $\mathbf{P}$. An element $A$ of $\mathscr{A}$ is said to be invariant if $T(A)=A$. We denote by $\mathscr{I}$ the $\sigma$-algebra of all invariant sets. The probability $\mathbf{P}$ is ergodic if each element of $\mathscr{I}$ has measure 0 or 1 .

Theorem 3. Let $\mathscr{M}_{0}$ be a sub- $\sigma$-algebra of $\mathscr{A}$ such that $\mathscr{M}_{0} \subseteq T^{-1}\left(\mathscr{M}_{0}\right)$ and define the nondecreasing filtration $\left(\mathscr{M}_{i}\right)_{i \in \mathbf{Z}}$ by $\mathscr{M}_{i}=T^{-i}\left(\mathscr{M}_{0}\right)$. Let $\mathbf{B}$ be a separable Banach space, and let $X_{0}$ be a random variable in $\mathbf{L}_{\mathbf{B}}^{1}$ with mean zero. Define the sequence $\left(X_{i}\right)_{i \in \mathbf{Z}}$ by $X_{i}=X_{0} \circ T^{i}$, and $S_{n}=X_{1}+\cdots+X_{n}$. For $1<p<2$, consider the condition

$$
G(p): \sum_{n=0}^{\infty} \mathbf{E}\left(X_{n} \mid \mathscr{M}_{0}\right) \text { and } \sum_{n=0}^{\infty}\left(X_{-n}-\mathbf{E}\left(X_{-n} \mid \mathscr{M}_{0}\right)\right) \text { converge in } \mathbf{L}_{\mathbf{B}}^{p} \text {. }
$$

If $G(p)$ holds and $\mathbf{B}$ is $r$-smooth for some $r>p$, then property (2.2) holds for any $1 \leqslant 1 / \alpha \leqslant p$.

$\mathrm{R}$ e $\mathrm{m}$ a $\mathrm{rk} 5$. For the strong law of large numbers (case $p=1$ ), no additional condition is needed. It follows from Mourier's ergodic theorem [18] that if $\mathbf{E}\left\|X_{0}\right\|_{\mathbf{B}}<\infty$, then

$$
\frac{1}{n} \sum_{k=1}^{n} X_{k} \text { converges almost surely to } \mathbf{E}\left(X_{0} \mid \mathscr{I}\right) \text {. }
$$

In fact, Mourier's result holds in any separable Banach space $\mathbf{B}$. Note that if $\mathbf{P}$ is ergodic and $\mathbf{B}=\mathbf{R}$, we cannot obtain property (2.3) without additional assumptions (see the example in [3, p. 117]).

$\mathrm{R}$ e $\mathrm{m}$ a $\mathrm{rk}$ 6. For the central limit theorem (case $p=2$ ), it is proved in [27] that: if $\mathbf{B}$ is 2-smooth and has a Schauder basis, if the $X_{i}$ of Theorem 3 are martingale differences with respect to the filtration $\left(\mathscr{M}_{i}\right)_{i \geqslant 0}$, and if $\mathbf{P}$ is ergodic, then

$$
\frac{1}{\sqrt{n}} \sum_{i=1}^{n} X_{i} \text { converges in distribution to a Gaussian measure on } \mathbf{B} \text {. }
$$

In fact (2.6) still holds if we replace the martingale assumption by condition $G(2)$ (this comes from the coboundary decomposition (2.7) below). For real-valued variables, this result is due to Gordin [11].

2.3. Proof of Theorem 3. It is based on a coboundary decomposition due to Gordin [11]. More precisely, according to Theorem 4.3 in [15], $G(p)$ holds if and only if

$$
X_{0}=M_{0}+Z_{0}-Z_{0} \circ T,
$$

where both $M_{0}$ and $Z_{0}$ belong to $\mathbf{L}_{\mathrm{B}}^{p}, M_{0}$ is $\mathscr{M}_{0}$ measurable and $\mathbf{E}\left(M_{0} \mid \mathscr{M}_{-1}\right)=0$ almost surely. Note that the result in [15] is given for real-valued variables. For Banach-valued variables, the proof is unchanged. 
Let $M_{i}=M_{0} \circ T^{i}$ and $Z_{i}=Z_{0} \circ T^{i}$. Note that $Q_{n}=M_{1}+\cdots+M_{n}$ is a martingale adapted to the filtration $\left(\mathscr{M}_{n}\right)_{n \geqslant 1}$. Clearly $S_{k}=Q_{k}+Z_{1}-Z_{k+1}$ and

$$
\max _{1 \leqslant k \leqslant n}\left\|S_{k}\right\|_{\mathbf{B}} \leqslant\left\|Z_{1}\right\|_{\mathbf{B}}+\max _{1 \leqslant k \leqslant n}\left\|Q_{k}\right\|_{\mathbf{B}}+\max _{2 \leqslant k \leqslant n+1}\left\|Z_{k}\right\|_{\mathbf{B}}
$$

According to Theorem 2, it suffices to prove that

$$
\sum_{n=1}^{\infty} n^{\alpha p-2} \mathbf{P}\left(\max _{2 \leqslant k \leqslant n+1}\left\|Z_{k}\right\|_{\mathbf{B}} \geqslant \varepsilon n^{\alpha}\right) \text { is finite. }
$$

By stationarity of $Z_{k}$, we infer that

$$
\sum_{n=1}^{\infty} n^{\alpha p-2} \mathbf{P}\left(\max _{2 \leqslant k \leqslant n+1}\left\|Z_{k}\right\|_{\mathbf{B}} \geqslant \varepsilon n^{\alpha}\right) \leqslant \sum_{n=1}^{\infty} n^{\alpha p-1} \mathbf{P}\left(\left\|Z_{0}\right\|_{\mathbf{B}} \geqslant \varepsilon n^{\alpha}\right) .
$$

Applying Fubini's theorem and using that $Z_{0}$ belongs to $\mathbf{L}_{\mathbf{B}}^{p}$, we infer that the right-hand term is finite, which completes the proof.

\section{The case of separable Hilbert spaces}

Condition $G(p)$ is expressed in terms of conditional expectations, so that it seems to be a reasonable extension of the martingale case. However, it does not lead to the optimal condition for strongly mixing sequences of real-valued variables, as we shall see in the sequel.

In this section, we obtain a sufficient condition for Hilbert-valued variables, which contains both the martingale case and the case of strongly mixing sequences. In order to develop our results, we need more definitions.

$\mathrm{D}$ e f i n it i o $\mathrm{n}$ 4. For any nonnegative integrable random variable $X$ with quantile function $Q_{X}$, let $H_{X}$ be the function $x \rightarrow \int_{0}^{x} Q_{X}(u) d u$. Note that, on the set $[0, \mathbf{P}(X>0)], H_{X}$ is an absolutely continuous and increasing function with values in $[0, \mathbf{E} X]$. Denote by $G_{X}$ the inverse of $H_{X}$.

$\mathrm{D}$ e f i $\mathrm{n}$ i t i o n 5 . Let $(\Omega, \mathscr{A}, \mathbf{P})$ be a probability space and $\left(\mathbf{B},\|\cdot\|_{\mathbf{B}}\right)$ a separable Banach space. For any sub- $\sigma$-algebra $\mathscr{M}$ of $\mathscr{A}$ and any random variable $Y$ in $\mathbf{L}_{\mathbf{B}}^{1}$, we consider the coefficient $\gamma(\mathscr{M}, Y)$ of weak dependence

$$
\gamma(\mathscr{M}, Y)=\|\mathbf{E}(Y \mid \mathscr{M})-\mathbf{E} Y\|_{\mathbf{L}_{\mathbf{B}}^{1}} .
$$

Denote by $\mathbf{P}_{Y}$ the distribution of $Y$ and by $\mathbf{P}_{Y \mid \mathscr{M}}$ a regular distribution of $Y$ given $\mathscr{M}$. Let $\sigma(Y)$ be the $\sigma$-algebra generated by the random variable $Y$. The strong mixing coefficient between $\mathscr{M}$ and $\sigma(Y)$ introduced in [24] may be defined as follows (see, for instance, [6, Proposition 3.22]):

$$
\alpha(\mathscr{M}, \sigma(Y))=\sup _{A \in \mathscr{B}(\mathbf{R})}\left\|\mathbf{P}_{Y \mid \mathscr{M}}(A)-\mathbf{P}_{Y}(A)\right\|_{1}
$$

(note that, with this definition, $\alpha\left(\mathscr{M}, \sigma\left(Y^{r}\right)\right)$ is two times the usual one). Let $\left(X_{i}\right)_{i>0}$ be a sequence of B-valued random variables, and let $\left(\mathscr{M}_{i}\right)_{i \geqslant 0}$ be 
a sequence of sub- $\sigma$-algebras of $\mathscr{A}$. The sequences of coefficients $\gamma_{i}$ and $\alpha_{i}$ are then defined by

$$
\gamma_{i}=\sup _{k \geqslant 0} \gamma\left(\mathscr{M}_{k}, X_{i+k}\right) \quad \text { and } \quad \alpha_{i}=\sup _{k \geqslant 0} \alpha\left(\mathscr{M}_{k}, \sigma\left(X_{i+k}\right)\right) .
$$

Let $\left(X_{i}\right)_{i \in \mathbf{Z}}$ and $\left(\mathscr{M}_{i}\right)_{i \in \mathbf{Z}}$ be defined as in Theorem 3 , and assume furthermore that $X_{0}$ is $\mathscr{M}_{0}$-measurable and in $\mathbf{L}_{\mathbf{H}}^{p}$ for some $p$ in $] 1,2[$. Let $X=\left\|X_{0}\right\|_{\mathbf{H}}$. We can prove (see Subsection 6.2 ) that condition $G(p)$ holds as soon as

$$
\sum_{i \geqslant 0}(i+1)^{p-1} \int_{0}^{\gamma_{i}} Q_{X}^{p-1} \circ G_{X}(u) d u<\infty .
$$

According to the inequality $2 G_{X}\left(\gamma_{k} / 18\right) \leqslant \alpha_{k}$, proved in [8, p. 250], we infer that (3.4) holds as soon as

$$
\sum_{i \geqslant 0}(i+1)^{p-1} \int_{0}^{\alpha_{i}} Q_{X}^{p}(u) d u<\infty .
$$

From [22], we know that, for real-valued variables, property (2.2) holds for $\alpha p=1$ and $p$ in $] 1,2[$ as soon as

$$
\sum_{i \geqslant 0}(i+1)^{p-2} \int_{0}^{\alpha_{i}} Q_{X}^{p}(u) d u<\infty
$$

which is clearly less restrictive than (3.5). Comparing (3.4) and (3.6), a natural question arises: does property (2.2) still holds for $\mathbf{H}$-valued random variables, under the condition

$$
D M(p, \gamma, X): \sum_{i \geqslant 0}(i+1)^{p-2} \int_{0}^{\gamma_{i}} Q_{X}^{p-1} \circ G_{X}(u) d u<\infty,
$$

which is implied by either (3.4) or (3.6)? We shall see in Theorem 4 below that the answer is positive, even in the nonstationary case. Note that in the stationary case the conditions $G(p)$ and $D M(p, \gamma, X)$ cannot be compared. However, it may happen that $G(p)$ does not hold for any $p$ in $] 1,2$ [, while $D M(p, \gamma, X)$ holds for any $p$ in $] 1,2$ [ (see again Subsection 6.2).

Theorem 4. Let $\left(X_{k}\right)_{k>0}$ be a sequence of random variables in $\mathbf{L}_{\mathbf{H}}^{1}$ and let $\mathscr{M}_{k}=\sigma\left(X_{i}, i \leqslant k\right)$. Let $S_{n}=\sum_{i=1}^{n}\left(X_{i}-\mathbf{E} X_{i}\right)$ and define the coefficients $\left(\gamma_{i}\right)_{i \geqslant 0}$ as in (3.3). Let $X$ be a positive random variable such that $\left(X_{i}\right) \prec X$.

1. If $\mathbf{E} X^{p}<\infty$ and $D M(p, \gamma, X)$ holds for some $p$ in $] 1,2[$, then (2.2) holds for any $1 \leqslant 1 / \alpha \leqslant p$.

2. If $\mathbf{E}\left(X \ln ^{+} X\right)<\infty$ and $\sum_{i \geqslant 1} \gamma_{i} / i<\infty$, then (2.3) holds.

$\mathrm{R}$ e $\mathrm{m}$ a $\mathrm{r} \mathrm{k} 7$. For a stationary sequence $\left(X_{i}\right)_{i \in \mathbf{Z}}$ of centered $\mathbf{H}$-valued random variables, the condition $D M\left(2, \gamma,\left\|X_{0}\right\|_{\mathbf{H}}\right)$ implies that $n^{-1 / 2} S_{n}$ converges weakly to a mixture of Gaussian distributions in $\mathbf{H}$. This result is proved in [8, Corollary 2]. 
From [7, Lemma 2], we obtain sufficient conditions for $D M(p, \gamma, X)$ to hold.

Corollary 1. Let $1<p \leqslant 2$. Any of the following conditions implies $D M(p, \gamma, X)$ :

1) $\mathbf{P}(X>x) \leqslant(c / x)^{r}$ for some $r>p$, and $\sum_{i \geqslant 0}(i+1)^{p-2} \times$ $\left(\gamma_{i}\right)^{(r-p) /(r-1)}<\infty$

2) $\|X\|_{r}<\infty$ for some $r>p$, and $\sum_{i \geqslant 1} i^{(p r-2 r+1) /(r-p)} \gamma_{i}<\infty$;

3) $\mathbf{E}\left(X^{p}(\ln (1+X))^{p-1}\right)<\infty$, and $\gamma_{i}=O\left(a^{i}\right)$ for some $a<1$.

If $\mathbf{H}=\mathbf{R}$, then the condition (3.6) may be weakened as shown in [22, Corollary 3.1]. For any real-valued random variable $Y$, let

$$
\left.\left.\left.\left.F_{Y}(t)=\mathbf{P}_{Y}(]-\infty, t\right]\right) \quad \text { and } \quad F_{Y \mid \mathscr{M}}(t)=\mathbf{P}_{Y \mid \mathscr{M}}(]-\infty, t\right]\right)
$$

Define

$$
\alpha(\mathscr{M}, Y)=\sup _{t \in \mathbf{R}}\left\|F_{Y \mid \mathscr{M}}(t)-F_{Y}(t)\right\|_{1} \quad \text { and } \quad \widetilde{\alpha}_{i}=\sup _{k>0} \alpha\left(\mathscr{M}_{k}, X_{i+k}\right) .
$$

From [9, Proposition 2, item 2], we know that, for real-valued variables, $G_{X}\left(\gamma_{k} / 2\right) \leqslant \widetilde{\alpha}_{k}$, so that $D M(p, \gamma, X)$ holds as soon as

$$
\sum_{i \geqslant 0}(i+1)^{p-2} \int_{0}^{\widetilde{\alpha}_{i}} Q_{X}^{p}(u) d u<\infty .
$$

Consequently, for $1<p<2$, condition $D M(p, \gamma, X)$ is weaker than Rio's criterion [22], [23]. For $p=1$, Rio does not assume that $\mathbf{E}\left(X \ln ^{+} X\right)$ is finite. In that case, the difference between our result and Rio's is the same as the difference between independent variables and martingale differences for the strong law of large numbers. Note also that Theorem 4 sharpens Theorem 1 in [26] in the special case where $p \in] 1,2[$. However the maximal inequality stated in Proposition 1 below does not allow one to obtain sharp results in the case where $1 \leqslant 1 / \alpha<2 \leqslant p<\infty$, which is also considered in [26].

To understand the difference between $\alpha_{i}$ and $\widetilde{\alpha}_{i}$, let us give the following example: if $\left(\epsilon_{i}\right)_{i>0}$ is i.i.d. with marginal $\operatorname{Bin}\left(\frac{1}{2}\right)$, then, for the stationary solution $\left(X_{i}\right)_{i>0}$ of the equation $2 X_{n}=X_{n-1}+\epsilon_{n}$, we have $\alpha_{i}=\frac{1}{2}$ and $\widetilde{\alpha}_{i} \leqslant 2^{-i}$.

Now to better understand the difference between $\widetilde{\alpha}_{i}$ and $\gamma_{i}$, note that one can build a sequence of martingale differences (i.e., $\gamma_{i}=0$ for $i>0$ ) such that $\widetilde{\alpha}_{i}$ does not converge to 0 . For the one-sided linear processes, $\gamma_{i}$ can easily be computed: let $\left(\epsilon_{i}\right)_{i \in \mathbf{Z}}$ be a stationary sequence of centered random variables in $\mathbf{L}_{\mathbf{H}}^{1},\left(a_{i}\right)_{i \geqslant 0}$ a sequence of linear operators from $\mathbf{H}$ to $\mathbf{H}$ such that $\sum_{i=0}^{\infty}\left\|a_{i}\right\|<\infty$, and $X_{n}=\sum_{i=0}^{\infty} a_{i}\left(\epsilon_{n-i}\right)$. For $i \geqslant 0$, let $\gamma_{i}^{\epsilon}=\| \mathbf{E}\left(\epsilon_{i} \mid \sigma\left(\epsilon_{j}\right.\right.$, $j \leqslant 0)) \|_{\mathbf{L}_{\mathbf{H}}^{1}}$ and $\gamma_{i}^{X}=\left\|\mathbf{E}\left(X_{i} \mid \sigma\left(X_{j}, j \leqslant 0\right)\right)\right\|_{\mathbf{L}_{\mathbf{H}}^{1}}$. We have the following upper bound:

$$
\gamma_{n}^{X} \leqslant \sum_{i=0}^{n-1}\left\|a_{i}\right\| \gamma_{n-i}^{\epsilon}+\left\|\epsilon_{0}\right\|_{\mathbf{L}_{\mathbf{H}}^{1}} \sum_{i=n}^{\infty}\left\|a_{i}\right\|
$$


Now, according to the inequality $G_{X}\left(\gamma_{k} / 2\right) \leqslant \widetilde{\alpha}_{k}$ and to the examples given in [22], we can see that condition $D M(p, \gamma, X)$ is essentially optimal. For instance, Corollary 2 below follows easily from Theorem 2 in [22] (apply the result of [21] with $a=r(p-1) /(r-p)$ and $b=r /(r-p))$.

Corollary 2. For any $1<p<2$ and any $r>p$, there exists a strictly stationary real-valued Markov chain $\left(X_{i}\right)_{i \in \mathbf{Z}}$ such that $\mathbf{E} X_{0}=0$ and

1) for any nonnegative real $x, \mathbf{P}\left(\left|X_{0}\right|>x\right)=\min \left(1, x^{-r}\right)$;

2) the sequence $\left(\gamma_{i}\right)_{i \geqslant 0}$ satisfies $\sup _{i>0} i^{p-1}(\ln i)\left(\gamma_{i}\right)^{(r-p) /(r-1)}<\infty$;

3) $\lim \sup _{n \rightarrow \infty}\left|S_{n}\right| / n^{1 / p}=+\infty$ almost surely.

We give now the maximal inequality (Proposition 1) which is the key result to prove Theorem 4. The proof of Proposition 1 will be done in Subsection 6.3 .

D e f i n i t i o n 6 . For any nonincreasing sequence $\left(\delta_{i}\right)_{i \geqslant 0}$ of nonnegative numbers, define $\delta^{-1}(u)=\sum_{i \geqslant 0} \mathbb{I}\left(u<\delta_{i}\right)=\inf \left\{k \in \mathbf{N}: \delta_{k} \leqslant u\right\}$. For any nonincreasing càdlàg function $f$, define the generalized inverse by $f^{-1}(u)=\inf \{t: f(t) \leqslant u\}$. Note that $\delta^{-1}$ is the generalized inverse of the càdlàg function $x \rightarrow \delta_{[x]},[\cdot]$ denoting the integer part.

Proposition 1. Let $\left(X_{k}\right)_{k>0}$ be a sequence of random variables in $\mathbf{L}_{\mathbf{H}}^{1}$ and let $\mathscr{M}_{k}=\sigma\left(X_{i}, i \leqslant k\right)$. Let $S_{n}=\sum_{i=1}^{n}\left(X_{i}-\mathbf{E} X_{i}\right)$ and define the coefficients $\left(\gamma_{i}\right)_{i \geqslant 0}$ as in (3.3). Let $X$ be a positive random variable such that $\left(X_{i}\right) \prec X$. Let $R_{X}=\left((\gamma / 2)^{-1} \circ G_{X}^{-1} \wedge n\right) Q_{X}$ and $S_{X}=R_{X}^{-1}$. For any $x>0$ and $r \geqslant 1$,

$$
\begin{aligned}
\mathbf{P}\left(\max _{1 \leqslant k \leqslant n}\left\|S_{k}\right\|_{\mathbf{H}} \geqslant 5 x\right) \leqslant & \frac{14 n}{x} \int_{0}^{S_{X}(x / r)} Q_{X}(u) d u \\
& +\frac{4 n}{x^{2}} \int_{S_{X}(x / r)}^{1} R_{X}(u) Q_{X}(u) d u .
\end{aligned}
$$

$\mathrm{R}$ e $\mathrm{m}$ a r k 8. Note that $\mathbf{E}|Z|^{p}=p \cdot 5^{p} \int_{0}^{\infty} x^{p-1} \mathbf{P}(|Z| \geqslant 5 x) d x$ for $p \geqslant 1$. With the same notation as in Proposition 1, we obtain from inequality (3.9) that, for any fixed real number $p$ in $] 1,2[$,

$$
\mathbf{E}\left(\max _{1 \leqslant k \leqslant n}\left\|S_{k}\right\|_{\mathbf{H}}^{p}\right) \leqslant C_{p} n \int_{0}^{1} R_{X}^{p-1}(u) Q_{X}(u) d u,
$$

where $C_{p}=5^{p} \cdot 2 p(12-5 p)(p-1)^{-1}(2-p)^{-1}$. By the definition of $R_{X}$, it follows that

$$
\mathbf{E}\left(\max _{1 \leqslant k \leqslant n}\left\|S_{k}\right\|_{\mathbf{H}}^{p}\right) \leqslant C_{p} n \sum_{i=0}^{n-1}(i+1)^{p-2} \int_{0}^{\gamma_{i} / 2} Q_{X}^{p-1} \circ G_{X}(u) d u .
$$

P r o of of The or e m 4. Proof of item 1. For the sake of brevity, write $L, Q, R, S$, and $G$ for $L_{X}, Q_{X}, R_{X}, S_{X}$, and $G_{X}$, respectively. Applying 
inequality (3.9) with $x=x_{n}=\left(\varepsilon n^{\alpha}\right) / 5$ and $r=1$, we obtain that, for any $\varepsilon \in] 0,1]$

$$
\begin{aligned}
n^{\alpha p-2} \mathbf{P}\left(\max _{1 \leqslant k \leqslant n}\left\|S_{k}\right\|_{\mathbf{H}} \geqslant \varepsilon n^{\alpha}\right) \leqslant & \frac{70}{\varepsilon} n^{\alpha(p-1)-1} \int_{0}^{S\left(x_{n}\right)} Q(u) d u \\
& +\frac{100}{\varepsilon^{2}} n^{\alpha(p-2)-1} \int_{S\left(x_{n}\right)}^{1} R(u) Q(u) d u .
\end{aligned}
$$

Since $R$ is right-continuous and nonincreasing,

$$
u<S\left(x_{n}\right) \Longleftrightarrow R(u)>\frac{\varepsilon n^{\alpha}}{5} \Longleftrightarrow n<\left(\frac{5 R(u)}{\varepsilon}\right)^{1 / \alpha} .
$$

Ending the proof as in [23, p. 59], we infer that there exists a finite constant $C$ depending only on $\alpha$ and on $\varepsilon$, such that for all $\varepsilon \in] 0,1]$,

$$
\begin{aligned}
\sum_{n \geqslant 1} n^{\alpha p-2} \mathbf{P}\left(\max _{1 \leqslant k \leqslant n}\left\|S_{k}\right\|_{\mathbf{H}} \geqslant \varepsilon n^{\alpha}\right) & \leqslant C \int_{0}^{1} R^{p-1}(u) Q(u) d u \\
& \leqslant C \int_{0}^{1}\left(\left(\frac{\gamma}{2}\right)^{-1} \circ G^{-1}(u)\right)^{p-1} Q^{p}(u) d u .
\end{aligned}
$$

Setting $v=H(u)$, the right-hand side is finite as soon as

$$
\int_{0}^{1}\left(\left(\frac{\gamma}{2}\right)^{-1}(u)\right)^{p-1} Q^{p-1} \circ G(u) d u<\infty
$$

which is equivalent to $D M(p, \gamma, X)$ (see, for instance, [23, Appendix $C]$ ).

Proof of item 2. We apply inequality (3.9) with $x=x_{n}=(\varepsilon n) / 5$ and $r=1$ :

$$
n^{-1} \mathbf{P}\left(\max _{1 \leqslant k \leqslant n}\left\|S_{k}\right\|_{\mathbf{H}} \geqslant \varepsilon n^{\alpha}\right) \leqslant I_{1}(n)+I_{2}(n)+I_{3}(n)
$$

where

$$
\begin{aligned}
& I_{1}(n)=\frac{70}{n \varepsilon} \int_{0}^{1} Q(u) \mathbb{I}\left(u<L\left(x_{n}\right)\right) d u, \\
& I_{2}(n)=\frac{70}{n \varepsilon} \int_{0}^{S\left(x_{n}\right)} Q(u) \mathbb{I}\left(u \geqslant L\left(x_{n}\right)\right) d u, \\
& I_{3}(n)=\frac{100}{\varepsilon^{2} n^{2}} \int_{S\left(x_{n}\right)}^{1} R(u) Q(u) d u .
\end{aligned}
$$

Note that, for any positive real $A$, we have

$$
Q_{X \mathbf{1}(X>A)}(u)=Q(u) \mathbb{I}(u<L(A)) .
$$

Applying first (3.13), we obtain that

$$
\frac{\varepsilon}{70} \sum_{n \geqslant 1} I_{1}(n)=\sum_{n \geqslant 1} n^{-1} \mathbf{E}\left(X \mathbb{I}\left(X>\frac{n \varepsilon}{5}\right)\right) .
$$


Then applying Fubini's theorem, it follows that $\sum_{n \geqslant 1} I_{1}(n)<\infty$ as soon as $\mathbf{E}\left(X \ln ^{+} X\right)<\infty$. On the other hand, using the fact that $L\left(x_{n}\right) \leqslant u<S\left(x_{n}\right)$ if and only if $Q(u) \leqslant x_{n}<R(u)$, we get

$$
\frac{\varepsilon}{70} \sum_{n \geqslant 1} I_{2}(n) \leqslant \int_{0}^{1} Q(u)\left(\sum_{1 \vee 5 Q(u) / \varepsilon \leqslant n<5 R(u) / \varepsilon} \frac{1}{n}\right) d u .
$$

Next, using the elementary inequalities $\ln (K+1)-\ln 2 \leqslant \sum_{n=2}^{K} n^{-1} \leqslant \ln K$, we easily infer that

$$
\frac{\varepsilon}{70} \sum_{n \geqslant 1} I_{2}(n) \leqslant \int_{0}^{1} Q(u) d u+\int_{0}^{1} Q(u) \ln \left(1+\left(\frac{\gamma}{2}\right)^{-1} \circ G^{-1}(u)\right) d u .
$$

Then, setting $v=H(u)$, it follows that

$$
\frac{\varepsilon}{70} \sum_{n \geqslant 1} I_{2}(n) \leqslant \mathbf{E}\|X\|_{\mathbf{H}}+\int_{0}^{\mathbf{E}\left\|_{X}\right\|_{\mathbf{H}}} \ln \left(1+\gamma^{-1}(u)\right) d u,
$$

and the right-hand side is finite if and only if $\sum_{i \geqslant 1} \gamma_{i} / i<\infty$.

Finally, using again (3.11), we obtain that

$$
\sum_{n \geqslant 1} n^{-2} \mathbb{I}\left(u \geqslant S\left(x_{n}\right)\right)=\sum_{n \geqslant 1 \vee 5 R(u) / \varepsilon} n^{-2} \leqslant 2\left(\frac{5 R(u)}{\varepsilon} \vee 1\right)^{-1} .
$$

Consequently, $\varepsilon^{2} \sum_{n \geqslant 1} I_{3}(n) \leqslant K \mathbf{E}\|X\|_{\mathbf{H}}$ for some $K$, which completes the proof.

\section{Cramér-von Mises statistics}

Let $(\Omega, \mathscr{A}, \mathbf{P}), T$, and $\mathscr{I}$ be as in Subsection 2.2. Let $X_{0}$ be a real-valued random variable and $X_{i}=X_{0} \circ T^{i}$. Let $F$ be the distribution function of $X_{0}$ and define

$$
F_{n}(t)=\frac{1}{n} \sum_{i=1}^{n} \mathbb{I}\left(X_{i} \leqslant t\right)
$$

Let $\mu$ be a $\sigma$-finite measure on $\mathbf{R}$ and suppose that $F$ satisfies

$$
\int_{\mathbf{R}_{-}}(F(t))^{2} \mu(d t)+\int_{\mathbf{R}_{+}}(1-F(t))^{2} \mu(d t)<\infty .
$$

Under this assumption, the process $\left\{t \rightarrow F_{n}(t)-F(t), t \in \mathbf{R}\right\}$ may be viewed as a random variable with values in the Hilbert space $\mathbf{L}^{2}(\mu)$. Define then

$$
D_{n}(\mu)=\left(\int\left(F_{n}(t)-F(t)\right)^{2} \mu(d t)\right)^{1 / 2} .
$$

When $\mu=d F, D_{n}(\mu)$ is known as the Cramér-von Mises statistics, and is commonly used for testing goodness of fit. It is'also interesting to write $D_{n}(\mu)$ as the supremum of the empirical process over a particular class of functions. For this task, we need the following lemma, whose proof will be done in Subsection 6.4. 
Lemma 1. For any two distributions functions $F$ and $G$ satisfying (4.1), define

$$
D(F, G, \mu)=\left(\int(F(t)-G(t))^{2} \mu(d t)\right)^{1 / 2} .
$$

Let $W_{1}(\mu)$ be the following set of functions:

$$
\begin{aligned}
\{f: f(t)= & f(0)+\left(\int_{[0, t]} g(x) \mu(d x)\right) \mathbb{I}(t>0) \\
& \left.-\left(\int_{[t, 0[} g(x) \mu(d x)\right) \mathbb{I}(t \leqslant 0), \quad \int(g(x))^{2} \mu(d x) \leqslant 1\right\} .
\end{aligned}
$$

Then we have that

$$
D(F, G, \mu)=\sup _{f \in W_{1}(\mu)}\left|\int f d F-\int f d G\right| .
$$

According to Lemma 1 , since $D_{n}(\mu)=D\left(F_{n}, F, \mu\right)$, we get that

$$
D_{n}(\mu)=\sup _{f \in W_{1}(\mu)}\left|\frac{1}{n} \sum_{i=1}^{n}\left(f\left(X_{i}\right)-\mathbf{E} f\left(X_{i}\right)\right)\right| .
$$

In particular, if $\mu$ is the Lebesgue measure on the real line, $W_{1}(\mu)$ contains the unit ball of the Sobolev space of order 1.

We now define the dependence coefficients which naturally appear in this context. Let $X$ be a real-valued random variable and $\mathscr{M}$ a sub- $\sigma$-algebra of $\mathscr{A}$. Keeping the same notation as in Definition 5, define the coefficient $\tau_{\mu}(\mathscr{M}, X)$ by

$$
\tau_{\mu}(\mathscr{M}, X)=\left\|\left(\int\left(F_{X \mid \mathscr{M}}(t)-F_{X}(t)\right)^{2} \mu(d t)\right)^{1 / 2}\right\|_{1} .
$$

The sequence $\left(\tau_{\mu}(i)\right)_{i \geqslant 0}$ of $\left(X_{i}\right)_{i \in \mathbf{Z}}$ is then defined by

$$
\tau_{\mu}(i)=\tau_{\mu}\left(\mathscr{M}_{0}, X_{i}\right) \quad \text { for } \quad \mathscr{M}_{0}=\sigma\left(X_{i}, i \leqslant 0\right) .
$$

With the help of this coefficient, we can describe the asymptotic behavior of $D_{n}(\mu)$.

Proposition 2. Assume that the distribution function $F$ of $X_{0}$ satisfies (4.1). Define the function $F_{\mu}$ by: $F_{\mu}(x)=\mu([0, x[)$ if $x \geqslant 0$ and $F_{\mu}(x)=-\mu\left(\left[x, 0[)\right.\right.$ if $x \leqslant 0$. Define also $Y_{\mu}=\sqrt{\left|F_{\mu}\left(X_{0}\right)\right|}$.

1. If $F_{X_{0} \mid \mathscr{I}}=F$ and $Y_{\mu}$ is integrable, then $D_{n}(\mu)$ converges to 0 almost surely.

2. If $D M\left(p, \tau_{\mu}, Y_{\mu}\right)$ holds for some $p$ in $] 1,2\left[\right.$, then $n^{(p-1) / p} D_{n}(\mu)$ converges to 0 almost surely.

3. If $D M\left(2, \tau_{\mu}, Y_{\mu}\right)$ holds, then $\sqrt{n} D_{n}(\mu)$ converges in distribution to $\left(\int G^{2}(x) \mu(d x)\right)^{1 / 2}$, where $G$ is a mixture of Gaussian processes. 
P r o o f. Define the variable $Z_{i}=\left\{t \rightarrow \mathbb{I}\left(X_{i} \leqslant t\right)-F(t), t \in \mathbf{R}\right\}$ which belongs to $\mathbf{H}=\mathbf{L}^{2}(\mu)$ as soon as (4.1) holds. Clearly

$$
\begin{aligned}
\left\|Z_{i}\right\|_{\mathbf{H}} \leqslant & \left(\int_{]-\infty, 0[}\left(\mathbb{I}\left(X_{i} \leqslant t\right)\right)^{2} \mu(d t)+\int_{[0, \infty[}\left(1-\mathbb{I}\left(X_{i} \leqslant t\right)\right)^{2} \mu(d t)\right)^{1 / 2} \\
& +\left(\int_{]-\infty, 0[}(F(t))^{2} \mu(d t)+\int_{[0, \infty[}(1-F(t))^{2} \mu(d t)\right)^{1 / 2}
\end{aligned}
$$

so that $\left\|Z_{i}\right\|_{\mathbf{H}} \leqslant \sqrt{\left|F_{\mu}\left(X_{i}\right)\right|}+\mathbf{E} \sqrt{\left|F_{\mu}\left(X_{i}\right)\right|}$ and $\mathbf{E}\left\|Z_{i}\right\|_{\mathbf{H}} \leqslant 2 \mathbf{E} Y_{\mu}$. Item 1 follows from (4.2) and Mourier's ergodic theorem, as quoted in Remark 5. Now, by definition of $\gamma_{k}$, we have that $\gamma_{k}=\mathbf{E}\left\|\mathbf{E}\left(Z_{k} \mid \mathscr{M}_{0}\right)\right\|_{\mathbf{H}}=\tau_{\mu}(k)$. On the other hand, we infer from (4.2) that

$$
Q_{\left\|Z_{i}\right\|_{\mathrm{H}}} \leqslant Q_{Y_{\mu}+\mathbf{E} Y_{\mu}} \leqslant Q_{Y_{\mu}}+\mathbf{E} Y_{\mu} .
$$

Since $\mathbf{E}\left\|Y_{\mu}\right\|_{\mathbf{H}} \leqslant \int_{0}^{1} Q_{Y_{\mu}}(u) d u$ and $Q_{Y_{\mu}}$ is nonincreasing, we get for all $x \in[0,1]$,

$$
\int_{0}^{x} Q_{\left\|Z_{0}\right\|_{\mathrm{H}}}(u) d u \leqslant \int_{0}^{x} Q_{Y_{\mu}}(u) d u+x \int_{0}^{1} Q_{Y_{\mu}}(u) d u \leqslant 2 \int_{0}^{x} Q_{Y_{\mu}}(u) d u .
$$

Now for two increasing continuous functions $f$ and $g$, we have that $f \leqslant g$ if and only if $f^{-1} \geqslant g^{-1}$. In addition, $[2 g(x)]^{-1}=g^{-1}(x / 2)$ and consequently $G_{\left\|Z_{0}\right\|_{\mathbf{H}}}(u) \geqslant G_{Y_{\mu}}(u / 2)$. From (4.3) and the last inequality we infer that, for any $1<p \leqslant 2$,

$$
\begin{aligned}
\int_{0}^{\gamma_{k}} Q_{\left\|Z_{0}\right\|_{\mathbf{H}}}^{p-1} \circ G_{\left\|Z_{0}\right\|_{\mathbf{H}}}(u) d u & \leqslant \int_{0}^{\gamma_{k}} Q_{Y_{\mu}}^{p-1} \circ G_{\left\|Z_{0}\right\|_{\mathbf{H}}}(u) d u+\int_{0}^{\gamma_{k}}\left(\mathbf{E} Y_{\mu}\right)^{p-1} d u \\
& \leqslant 2 \int_{0}^{\gamma_{k} / 2} Q_{Y_{\mu}}^{p-1} \circ G_{Y_{\mu}}(u) d u+\gamma_{k}\left(\mathbf{E} Y_{\mu}\right)^{p-1} .
\end{aligned}
$$

Since $\gamma_{k}=\tau_{\mu}(k)$, we infer from (4.5) that $D M\left(p, \gamma,\left\|Z_{0}\right\|_{\mathbf{H}}\right)$ holds as soon as $D M\left(p, \tau_{\mu}, Y_{\mu}\right)$ does. Hence item 2 follows from Theorem 4 (together with Remark 1) and item 3 from [8, Corollary 2] (the covariance structure of $G$ conditionally to $\mathscr{I}$ is given in Example 2 of the same paper).

4.1. Examples. The coefficients $\tau_{\mu}(\mathscr{M}, X)$ may be compared to $\alpha(\mathscr{M}, \sigma(X)), \alpha(\mathscr{M}, X)$ and to other dependence coefficients introduced in [9]). Define $\tau(\mathscr{M}, X)$ and $\beta(\mathscr{M}, X)$ by

$$
\begin{aligned}
& \tau(\mathscr{M}, X)=\int\left\|F_{X \mid \mathscr{M}}(t)-F_{X}(t)\right\|_{1} d t, \\
& \beta(\mathscr{M}, X)=\left\|\sup _{t \in \mathbf{R}}\left|F_{X \mid \mathscr{M}}(t)-F_{X}(t)\right|\right\|_{1} .
\end{aligned}
$$

Lemma 2. For any real random variable $X$ and any $\sigma$-algebra $\mathscr{M}$, we have: 
1) Let $Y_{\mu}=\sqrt{\left|F_{\mu}(X)\right|}$; then

$$
\tau_{\mu}(\mathscr{M}, X) \leqslant 36 \int_{0}^{\alpha(\mathscr{M}, \sigma(X)) / 2} Q_{Y_{\mu}}(u) d u ;
$$

2) if $\mu$ is a probability measure, then $\tau_{\mu}(\mathscr{M}, X) \leqslant \sqrt{\alpha(\mathscr{M}, X)}$ and $\tau_{\mu}(\mathscr{M}, X) \leqslant \beta(\mathscr{M}, X)$

3) if $F_{\mu}$ is $K$-Lipschitz, then $\tau_{\mu}(\mathscr{M}, X) \leqslant \sqrt{K \tau(\mathscr{M}, X)}$.

Recall that $\tau(\mathscr{M}, X)$ has the following property: if $X^{*}$ is any random variable independent of $\mathscr{M}$ and distributed as $X$, then $\tau(\mathscr{M}, X) \leqslant$ $\left\|X-X^{*}\right\|_{1}$. Further, if $\Omega$ is rich enough, one can choose $X^{*}$ such that $\tau(\mathscr{M}, X)=\left\|X-X^{*}\right\|_{1}$. Due to this property, the coefficient $\tau$ is easy to compute in many situations. Among the large variety of examples given in [9], let us choose two important cases: if $X_{n}=\sum_{j \geqslant 0} a_{j} \xi_{n-j}$, where $\left(\xi_{i}\right)_{i \in \mathbf{Z}}$ is i.i.d., then $\tau\left(\mathscr{M}_{0}, X_{n}\right) \leqslant 2\left\|\xi_{0}\right\|_{1}\left(\sum_{j \geqslant n}\left|a_{j}\right|\right)$ and also $\tau\left(\mathscr{M}_{0}, X_{n}\right) \leqslant\left(2 \mathbf{D}\left(\xi_{0}\right) \sum_{j \geqslant n} a_{j}^{2}\right)^{1 / 2}$. If $\left(X_{n}\right)_{n \geqslant 0}$ is the stationary solution of the equation $X_{n}=f\left(X_{n-1}\right)+\xi_{n}$, where $f$ is $\kappa$-Lipschitz for some $\kappa<1$ and $\left(\xi_{i}\right)_{i \in \mathbf{Z}}$ is i.i.d., then $\tau\left(\mathscr{M}_{0}, X_{n}\right) \leqslant 2\left\|X_{0}\right\|_{1} \kappa^{n}$. Note also that the coefficient $\beta(\mathscr{M}, X)$ is well adapted to dynamical systems (see [9, Section 4.4]).

P r o of of $\mathrm{L} \mathrm{e} \mathrm{m} \mathrm{m} \mathrm{a} \mathrm{2.} \mathrm{Define} \mathrm{the} \mathrm{variable} Z=\{t \rightarrow \mathbb{I}(X \leqslant t)-$ $F(t), t \in \mathbf{R}\}$ which belongs to $\mathbf{H}=\mathbf{L}^{2}(\mu)$ as soon as (4.1) holds. Using the definition of $\tau(\mathscr{M}, X)$ and an inequality of [8] (stated at the end of the proof of Corollary 2 in [8]), we infer that

$$
\tau_{\mu}(\mathscr{M}, X)=\mathbf{E}\|\mathbf{E}(Z \mid \mathscr{M})\|_{\mathbf{H}} \leqslant 18 \int_{0}^{\alpha(\mathscr{M}, \sigma(X)) / 2} Q_{\|Z\|_{\mathbf{H}}}(u) d u .
$$

Now, it follows from (4.3) that $Q_{\|Z\|_{\mathbf{H}}} \leqslant Q_{Y_{\mu}}+\mathbf{E} Y_{\mu}$. Since $Q_{Y_{\mu}}$ is nonincreasing, item 1 follows from (4.4) and (4.6).

If $\mu$ is a probability measure, then

$$
\tau_{\mu}(\mathscr{M}, X) \leqslant\left(\int\left\|F_{X \mid \mathscr{M}}(t)-F_{X}(t)\right\|_{1} \mu(d t)\right)^{1 / 2} \leqslant \sqrt{\alpha(\mathscr{M}, X)} .
$$

The second inequality of item 2 follows from the bound

$$
\tau_{\mu}(\mathscr{M}, X) \leqslant\left\|\left(\int\left(\sup _{t \in \mathbf{R}}\left|F_{X \mid \mathscr{M}}(t)-F_{X}(t)\right|\right)^{2} \mu(d t)\right)^{1 / 2}\right\|_{1}=\beta(\mathscr{M}, X) .
$$

We now prove item 3 . Since $F_{\mu}$ is $K$-Lipschitz, $\mu$ is absolutely continuous with respect to the Lebesgue measure, with a density bounded by $K$. Consequently,

$$
\tau_{\mu}(\mathscr{M}, X) \leqslant\left(K \int\left\|F_{X \mid \mathscr{M}}(t)-F_{X}(t)\right\|_{1} d t\right)^{1 / 2}=\sqrt{K \tau(\mathscr{M}, X)} .
$$




\section{Estimation of the covariance operator of a Hilbert autoregressive process}

Let $\mathbf{H}$ be a separable Hilbert space with norm $\|\cdot\|_{\mathbf{H}}$ and inner product $\langle\cdot, \cdot\rangle_{\mathbf{H}}$. Denote by $L(\mathbf{H})$ the class of bounded linear operators from $\mathbf{H}$ to $\mathbf{H}$ and by $\|\cdot\|_{L(\mathbf{H})}$ the usual norm on $L(\mathbf{H})$. A strictly stationary $\mathbf{H}$-valued process $X=\left(X_{n}\right)_{n \in \mathbf{Z}}$ is an autoregressive process of order $1(\mathrm{ARH}(1))$ if it satisfies

$$
X_{n}-\mu=\rho\left(X_{n-1}-\mu\right)+\xi_{n}, \quad n \in \mathbf{Z},
$$

where $\mu \in \mathbf{H}, \rho \in L(\mathbf{H})$ and $\left(\xi_{n}\right)_{n \in \mathbf{Z}}$ is a strictly stationary sequence of centered random variables in $\mathbf{L}_{\mathbf{H}}^{1}$. The existence of such a process is ensured by the mild condition (see [5])

there exists an integer $j_{0} \geqslant 1$ such that $\left\|\rho^{j_{0}}\right\|_{L(\mathbf{H})}<1$.

Indeed, if (5.2) holds, then (5.1) has a unique stationary solution given by

$$
X_{n}=\mu+\sum_{j \geqslant 0} \rho^{j}\left(\xi_{n-j}\right), \quad n \in \mathbf{Z},
$$

where the series is convergent almost surely and in $\mathbf{L}_{\mathbf{H}}^{1}$. These types of processes with values in functional spaces facilitate the study of estimation and forecasting problems for several classes of continuous time processes. For more details we refer to [5].

Consider an $\operatorname{ARH}(1) X=\left(X_{n}\right)_{n \in \mathbf{Z}}$ generated by a sequence $\xi=\left(\xi_{n}\right)_{n \in \mathbf{Z}}$ of i.i.d. random variables in $\mathbf{L}_{\mathbf{H}}^{2}$. Define the covariance operator $C_{X_{0}}$ of $X_{0}$ by

$$
C_{X_{0}}(x)=\mathbf{E}\left(\left\langle X_{0}, x\right\rangle_{\mathbf{H}} X_{0}\right), \quad x \in \mathbf{H} .
$$

The natural estimator of $C_{X_{0}}$ is the empirical covariance operator, defined by $C_{n}(x)=n^{-1} \sum_{i=1}^{n}\left\langle X_{i}, x\right\rangle_{\mathbf{H}} X_{i}, x \in \mathbf{H}$. We wish to study the behavior of the random variable $C_{n}-C_{X_{0}}$ with values in a certain functional space. Such results are useful for the estimation of the eigenvalues of $C_{X_{0}}$ which allows one to derive results on the estimation of $\rho$ (see [5, Chapter 8]). The main tool for studying $C_{n}$ is an autoregressive representation. Set

$$
Z_{i}(x)=\left\langle X_{i}, x\right\rangle_{\mathbf{H}} X_{i}-C_{X_{0}}(x), \quad x \in \mathbf{H}, \quad i \in \mathbf{Z} .
$$

Let $\mathscr{S}$ be the space of Hilbert-Schmidt operators on $\mathbf{H}$, that is, the space of bounded linear operators $s$ from $\mathbf{H}$ to $\mathbf{H}$ such that the quantity $\|s\|_{\mathscr{S}}=$ $\left(\sum_{1 \leqslant i \leqslant \infty}\left\|s\left(g_{i}\right)\right\|_{\mathbf{H}}^{2}\right)^{1 / 2}$ is finite for any orthonormal basis $\left(g_{i}\right)_{i \geqslant 1}$ of $\mathbf{H}$. This is a separable Hilbert space with respect to the scalar product

$$
\left\langle s_{1}, s_{2}\right\rangle_{\mathscr{S}}=\sum_{1 \leqslant i, j \leqslant \infty}\left\langle s_{1}\left(g_{i}\right), h_{j}\right\rangle_{\mathbf{H}}\left\langle s_{2}\left(g_{i}\right), h_{j}\right\rangle_{\mathbf{H}},
$$

where $\left(g_{i}\right)_{i \geqslant 1}$ and $\left(h_{j}\right)_{j \geqslant 1}$ are two arbitrary orthonormal bases of $\mathbf{H}$. According to [5, Lemma 4.1], the $\mathscr{S}$-valued process $Z=\left(Z_{n}\right)_{n \in \mathbf{Z}}$ admits the following $\mathrm{AR} \mathscr{S}(1)$ representation:

$$
Z_{i}=R\left(Z_{i-1}\right)+E_{i}, \quad i \in \mathbf{Z},
$$


where $R \in L(\mathscr{S})$ is defined by $R(s)=\rho s \rho^{*}$ for all $s \in \mathscr{S}$ and satisfies $\left\|R^{h}\right\|_{L(\mathscr{S})} \leqslant\left\|\rho^{h}\right\|_{L(\mathbf{H})}^{2}$, and $E=\left(E_{n}\right)_{n \in \mathbf{Z}}$ is a sequence of $\mathscr{S}$-valued martingale differences with respect to the filtration $\left(\sigma\left(\xi_{k}, k \leqslant n\right)\right)$. Note that $E_{n}$ belongs to $\mathbf{L}_{\mathscr{S}}^{p}$ as soon as $\xi$ belongs to $\mathbf{L}_{\mathbf{H}}^{2 p}$. Concerning the behavior of the random variable $\left\|C_{n}-C_{X_{0}}\right\|_{\mathscr{S}}$, the following results hold.

Proposition 3. Let $\rho$ be an operator of $L(\mathbf{H})$ satisfying (5.2). Assume that $\left(\xi_{n}\right)_{n \in \mathbf{Z}}$ is an i.i.d. sequence of random variables in $\mathbf{L}_{\mathbf{H}}^{2}$. Let $\left(X_{n}\right)_{n \in \mathbf{Z}}$ be the strictly stationary solution of (5.1).

1. The sequence $\left\|C_{n}-C_{X_{0}}\right\|_{\mathscr{S}}$ converges almost surely to 0 .

2. If $\mathbf{E}\left\|\xi_{0}\right\|_{\mathbf{H}}^{2 p}<\infty$ for some $p$ in $] 1,2\left[\right.$, then $n^{(p-1) / p}\left\|C_{n}-C_{X_{0}}\right\|_{\mathscr{S}}$ converges almost surely to 0 .

3. If $\mathbf{E}\left\|\xi_{0}\right\|_{\mathbf{H}}^{4}<\infty$, then $\sqrt{n}\left\|C_{n}-C_{X_{0}}\right\|_{\mathscr{S}}$ converges in distribution to $\|G\|_{\mathscr{S}}$, where $G$ is an $\mathscr{S}$-valued centered Gaussian random variable.

$\mathrm{R}$ e $\mathrm{m}$ a $\mathrm{r} \mathrm{k}$ 9. Item 3 has been proved in [5, Corollary 4.6] and can be proved also by noting that condition $G(2)$ is satisfied (see Remark 6). The covariance operator of the $\mathscr{S}$-valued Gaussian random variable $G$ is given in [5].

Proof of Proposition 3. The fact that $\left(\xi_{n}\right)_{n \in \mathbf{Z}}$ is an i.i.d. sequence ensures the ergodicity of the sequence $\left(Z_{n}\right)_{n \in \mathbf{Z}}$ defined by (5.3). Hence, item 1 follows from Mourier's ergodic theorem [18] as quoted in Remark 5. To prove item 2, note that $E_{0}$ belongs to $\mathbf{L}_{\mathscr{S}}^{p}$ and that

$$
\left\|\mathbf{E}\left(Z_{n} \mid \mathscr{M}_{0}\right)\right\|_{\mathbf{L}_{\mathscr{S}}^{p}} \leqslant\left\|E_{0}\right\|_{\mathbf{L}_{\mathscr{S}}^{p}} \sum_{j \geqslant n}\left\|R^{j}\right\|_{L(\mathscr{S})} .
$$

Since (5.2) is satisfied and $\left\|R^{j}\right\|_{L(\mathscr{S})} \leqslant\left\|\rho^{j}\right\|_{L(\mathbf{H})}^{2}$, we infer that condition $G(p)$ of Theorem 3 holds. This completes the proof.

\section{Appendix}

6.1. Proof of Theorem 2. Proof of item 1. Define the four variables

$$
\begin{aligned}
& X_{i}^{\prime}=X_{i} \mathbb{I}\left(\left\|X_{i}\right\|_{\mathbf{B}} \leqslant n^{\alpha}\right) \quad \text { and } \quad X_{i}^{\prime \prime}=X_{i} \mathbb{I}\left(\left\|X_{i}\right\|_{\mathbf{B}}>n^{\alpha}\right), \\
& Y_{i}^{\prime}=X_{i}^{\prime}-\mathbf{E}\left(X_{i}^{\prime} \mid \mathscr{M}_{i-1}\right) \quad \text { and } \quad Y_{i}^{\prime \prime}=X_{i}^{\prime \prime}-\mathbf{E}\left(X_{i}^{\prime \prime} \mid \mathscr{M}_{i-1}\right) .
\end{aligned}
$$

Since $\left(X_{i}\right)_{i \geqslant 1}$ is a sequence of martingale differences, it follows that $X_{i}=$ $Y_{i}^{\prime}+Y_{i}^{\prime \prime}$. Hence, for every positive $\varepsilon$,

$$
\begin{aligned}
\mathbf{P}\left(\max _{1 \leqslant k \leqslant n}\left\|S_{k}\right\|_{\mathbf{B}} \geqslant 2 \varepsilon n^{\alpha}\right) \leqslant & \mathbf{P}\left(\max _{1 \leqslant k \leqslant n}\left\|\sum_{j=1}^{k} Y_{j}^{\prime}\right\|_{\mathbf{B}} \geqslant \varepsilon n^{\alpha}\right) \\
& +\mathbf{P}\left(\max _{1 \leqslant k \leqslant n}\left\|\sum_{j=1}^{k} Y_{j}^{\prime \prime}\right\|_{\mathbf{B}} \geqslant \varepsilon n^{\alpha}\right) .
\end{aligned}
$$


Applying Markov's inequality, we obtain that

$$
\begin{aligned}
\mathbf{P}\left(\max _{1 \leqslant k \leqslant n}\left\|\sum_{j=1}^{k} Y_{j}^{\prime \prime}\right\|_{\mathbf{B}} \geqslant \varepsilon n^{\alpha}\right) & \leqslant \frac{2}{\varepsilon n^{\alpha}} \sum_{k=1}^{n} \mathbf{E}\left\|X_{k}^{\prime \prime}\right\|_{\mathbf{B}} \\
& =\frac{2}{\varepsilon n^{\alpha}} \sum_{k=1}^{n} \mathbf{E}\left(\left\|X_{k}\right\|_{\mathbf{B}} \mathbb{I}\left(\left\|X_{k}\right\|_{\mathbf{B}}>n^{\alpha}\right)\right) .
\end{aligned}
$$

Note that for any $k \geqslant 1$,

$$
\begin{aligned}
\mathbf{E}\left(\left\|X_{k}\right\|_{\mathbf{B}} \mathbb{I}\left(\left\|X_{k}\right\|_{\mathbf{B}}>n^{\alpha}\right)\right) & =\int_{0}^{1} Q_{\left\|X_{k}\right\|_{\mathbf{B}} \mathbf{I}\left(\left\|X_{k}\right\|_{\mathbf{B}}>n^{\alpha}\right)}(u) d u \\
& \leqslant \int_{0}^{1} Q_{X \mathbf{I}\left(X>n^{\alpha}\right)}(u) d u .
\end{aligned}
$$

For simplicity, let $Q=Q_{X}$ and $L=L_{X}$. From (6.2), (6.3), and (3.13), we infer that

$$
\mathbf{P}\left(\max _{1 \leqslant k \leqslant n}\left\|\sum_{j=1}^{k} Y_{j}^{\prime \prime}\right\|_{\mathbf{B}} \geqslant \varepsilon n^{\alpha}\right) \leqslant \frac{2 n^{1-\alpha}}{\varepsilon} \int_{0}^{1} Q(u) \mathbb{I}\left(u<L\left(n^{\alpha}\right)\right) d u .
$$

Since $u<L\left(n^{\alpha}\right)$ if and only if $n<Q^{1 / \alpha}(u)$, we infer that there exists a finite constant $C$ depending only on $p$ and $\alpha$ such that

$$
\begin{aligned}
\sum_{n \geqslant 1} n^{\alpha p-2} \mathbf{P}\left(\max _{1 \leqslant k \leqslant n}\left\|\sum_{j=1}^{k} Y_{j}^{\prime \prime}\right\|_{\mathbf{B}} \geqslant \varepsilon n^{\alpha}\right) & \leqslant \frac{2}{\varepsilon} \int_{0}^{1} Q(u)\left(\sum_{n=1}^{\left[Q^{1 / \alpha}(u)\right]} n^{\alpha(p-1)-1}\right) d u \\
& \leqslant C \int_{0}^{1} Q^{p}(u) d u=C \mathbf{E} X^{p} .
\end{aligned}
$$

It remains to control the first term on the right-hand side in (6.1). Applying Doob's inequality to the submartingale $\left\|\sum_{j=1}^{k} Y_{j}^{\prime}\right\|_{\mathrm{B}}$ we have that

$$
\mathbf{P}\left(\max _{1 \leqslant k \leqslant n}\left\|\sum_{j=1}^{k} Y_{j}^{\prime}\right\|_{\mathbf{B}} \geqslant \varepsilon n^{\alpha}\right) \leqslant \frac{1}{\varepsilon^{r} n^{r \alpha}} \mathbf{E}\left\|\sum_{j=1}^{n} Y_{j}^{\prime}\right\|_{\mathbf{B}}^{r} .
$$

Since $\mathbf{B}$ is $(r, D)$-smooth, we obtain, applying inequality (2.1)

$$
\mathbf{P}\left(\max _{1 \leqslant k \leqslant n}\left\|\sum_{j=1}^{k} Y_{j}^{\prime}\right\|_{\mathbf{B}} \geqslant \varepsilon n^{\alpha}\right) \leqslant \frac{D}{\varepsilon^{r} n^{r \alpha}} \sum_{j=1}^{n} \mathbf{E}\left\|Y_{j}^{\prime}\right\|_{\mathbf{B}}^{r} \leqslant \frac{2^{r} D}{\varepsilon^{r} n^{r \alpha}} \sum_{j=1}^{n} \mathbf{E}\left\|X_{j}^{\prime}\right\|_{\mathbf{B}}^{r} .
$$

Bearing in mind the definition of $X_{j}^{\prime}$ we infer that

$$
\mathbf{P}\left(\max _{1 \leqslant k \leqslant n}\left\|\sum_{j=1}^{k} Y_{j}^{\prime}\right\|_{\mathbf{B}} \geqslant \varepsilon n^{\alpha}\right) \leqslant \frac{2^{r} D}{\varepsilon^{r} n^{r \alpha}} \sum_{i=1}^{n} \int_{0}^{1} Q_{\left\|X_{i}\right\|_{\mathbf{B}} \mathbf{I}\left(\left\|X_{i}\right\|_{\mathbf{B}} \leqslant n^{\alpha}\right)}(u) d u .
$$

Note that for any $A>0$ and any $k \geqslant 1,\left\|X_{k}\right\|_{\mathbf{B}} \mathbb{I}\left(\left\|X_{k}\right\|_{\mathbf{B}} \leqslant A\right) \leqslant\left\|X_{k}\right\|_{\mathbf{B}} \wedge A$. Now, for any $u \in[0,1]$,

$$
Q_{\left\|X_{k}\right\|_{\mathbf{B}} \mathbf{I}\left(\left\|X_{k}\right\|_{\mathbf{B}} \leqslant A\right)}(u) \leqslant Q_{\left\|X_{k}\right\|_{\mathbf{B}} \wedge A}(u) \leqslant Q_{X \wedge A}(u) \leqslant Q(u) \vee A .
$$


From (6.6), (6.7) and the fact that $A<Q(u)$ if and only if $u<L(A)$, we infer that

$$
\sum_{n \geqslant 1} n^{\alpha p-2} \mathbf{P}\left(\max _{1 \leqslant k \leqslant n}\left\|\sum_{j=1}^{k} Y_{j}^{\prime}\right\|_{\mathbf{B}} \geqslant \varepsilon n^{\alpha}\right) \leqslant \frac{2^{r} D}{\varepsilon^{r}}\left(A_{1}(n)+A_{2}(n)\right),
$$

where

$$
\begin{aligned}
& A_{1}(n)=\sum_{n \geqslant 1} n^{\alpha(p+1-r)-1} \int_{0}^{1} Q^{r-1}(u) \mathbb{I}\left(u<L\left(n^{\alpha}\right)\right) d u, \\
& A_{2}(n)=\sum_{n \geqslant 1} n^{\alpha(p-r)-1} \int_{0}^{1} Q^{r}(u) \mathbb{I}\left(L\left(n^{\alpha}\right) \leqslant u \leqslant 1\right) d u .
\end{aligned}
$$

Now, since $u \geqslant L\left(n^{\alpha}\right)$ if and only if $n \geqslant Q^{1 / \alpha}(u)$, there exists two finite constants $K_{1}$ and $K_{2}$ depending only on $\alpha, p$ and $r$, such that

$$
\begin{aligned}
& A_{1}(n) \leqslant \int_{0}^{1} Q^{r-1}(u)\left(\sum_{n=1}^{\left[Q^{1 / \alpha}(u)\right]} n^{\alpha(p+1-r)-1}\right) d u \leqslant K_{1} \int_{0}^{1} Q^{p}(u) d u, \\
& A_{2}(n) \leqslant \int_{0}^{1} Q^{r}(u)\left(\sum_{n \geqslant Q^{1 / \alpha}(u)} n^{\alpha(p-r)-1}\right) d u \leqslant K_{2} \int_{0}^{1} Q^{p}(u) d u .
\end{aligned}
$$

Finally, $A_{1}(n)$ and $A_{2}(n)$ are finite as soon as $\mathbf{E} X^{p}$ is finite, which completes the proof.

Proof of item 2. Since B is super-reflexive, it is $r$-smooth for some $1<r \leqslant 2$. Without loss of generality, assume that $r<2$. According to inequality (6.3), for all $k \geqslant 1$,

$$
\mathbf{E}\left(\left\|X_{k}\right\|_{\mathbf{B}} \mathbb{I}\left(\left\|X_{k}\right\|_{\mathbf{B}}>n\right)\right) \leqslant \mathbf{E}(X \mathbb{I}(X>n)) .
$$

Consequently, by applying Fubini's theorem, we infer from (6.2) that if $\mathbf{E}\left(X \ln ^{+} X\right)<\infty$, then

$$
\sum_{n \geqslant 1} \frac{1}{n} \mathbf{P}\left(\max _{1 \leqslant k \leqslant n}\left\|\sum_{j=1}^{k} Y_{j}^{\prime \prime}\right\|_{\mathbf{B}} \geqslant \varepsilon n\right)<\infty .
$$

It remains to prove that $\sum_{n \geqslant 1} n^{-1} \mathbf{P}\left(\max _{1 \leqslant k \leqslant n}\left\|\sum_{j=1}^{k} Y_{j}^{\prime}\right\|_{\mathbf{B}} \geqslant \varepsilon n\right)<\infty$. Starting from (6.8) with $\alpha=p=1$ and applying inequalities (6.9) and (6.10), we infer that $A_{1}(n)$ and $A_{2}(n)$ are finite as soon as $\mathbf{E} X$ is finite, which completes the proof.

6.2. Comparison of the conditions $G(p),(3.4)$, and $D M(p, \gamma, X)$. In this section, we consider a stationary sequence $\left(X_{i}\right)_{i \in \mathbf{Z}}$ with values in $\mathbf{L}_{\mathbf{H}}^{p}$ with $\left.\left.p \in\right] 1,2\right]$, such that $X_{0}$ is centered and $\mathscr{M}_{0}$-measurable. Let $X=\left\|X_{0}\right\|_{\mathbf{H}}$. 
Proof of the implication $(3.4) \Rightarrow G(p)$. Notice first that $G(p)$ can be rewritten as

$$
\lim _{m \rightarrow \infty} \sup _{n>m}\left\|\mathbf{E}\left(\sum_{i=m+1}^{n} X_{i} \mid \mathscr{M}_{0}\right)\right\|_{\mathbf{L}_{\mathbf{H}}^{p}}=0 .
$$

Since $p>1$, using first the duality between $\mathbf{L}_{\mathbf{H}}^{p}$ and $\mathbf{L}_{\mathbf{H}}^{q}$ for $q=(p-1) / p$, followed by the inequality (3.29) in [8], we successively have

$$
\begin{aligned}
\left\|\mathbf{E}\left(\sum_{i=m+1}^{n} X_{i} \mid \mathscr{M}_{0}\right)\right\|_{\mathbf{L}_{\mathbf{H}}^{p}} & =\sup _{Y \text { is } \mathscr{M}_{0} \text {-measurable, }}^{\|Y\|_{\mathbf{L}_{\mathbf{H}}^{q}} \leqslant 1} \in \mathbf{E}\left\langle Y, \sum_{i=m+1}^{n} X_{i}\right\rangle_{\mathbf{H}} \\
& \leqslant \sup _{\|Y\|_{\mathbf{L}_{\mathbf{H}}^{q}} \leqslant 1} \sum_{i=m+1}^{n} \int_{0}^{1} Q_{Y}(u) Q_{X}(u) \mathbb{I}_{u \leqslant G_{X}\left(\gamma_{i}\right)} d u .
\end{aligned}
$$

Then Hölder's inequality yields

$$
\begin{aligned}
\left\|\mathbf{E}\left(\sum_{i=m+1}^{n} X_{i} \mid \mathscr{M}_{0}\right)\right\|_{\mathbf{L}_{\mathbf{H}}^{p}} \leqslant & \sup _{\|Y\|_{\mathbf{L}_{\mathbf{H}}^{q}} \leqslant 1}\left(\int_{0}^{1} Q_{Y}^{q}(u) d u\right)^{1 / q} \\
& \times\left(\int_{0}^{1} Q_{X}^{p}(u)\left(\sum_{i=m+1}^{n} \mathbb{I}\left(u \leqslant G_{X}\left(\gamma_{i}\right)\right)\right)^{p} d u\right)^{1 / p} .
\end{aligned}
$$

Since

$$
\left(\sum_{i=m+1}^{\infty} \mathbb{I}\left(u \leqslant G_{X}\left(\gamma_{i}\right)\right)\right)^{p} \leqslant \sum_{j=m+1}^{\infty}(j+1)^{p} \mathbb{I}\left(G_{X}\left(\gamma_{j+1}\right) \leqslant u \leqslant G_{X}\left(\gamma_{j}\right)\right)
$$

and since, for $1<p \leqslant 2,(j+1)^{p} \leqslant 2 \sum_{\ell=0}^{j}(\ell+1)^{p-1}$, we get

$$
\begin{aligned}
& \left(\sum_{i=m+1}^{\infty} \mathbb{I}\left(u \leqslant G_{X}\left(\gamma_{i}\right)\right)\right)^{p} \\
& \quad \leqslant 2 \sum_{j=m+1}^{\infty}\left(\sum_{\ell=0}^{j}(\ell+1)^{p-1}\right) \mathbb{I}\left(G_{X}\left(\gamma_{j+1}\right) \leqslant u \leqslant G_{X}\left(\gamma_{j}\right)\right) .
\end{aligned}
$$

Then

$$
\begin{aligned}
\left\|\mathbf{E}\left(\sum_{i=m+1}^{n} X_{i} \mid \mathscr{M}_{0}\right)\right\|_{\mathbf{L}_{\mathbf{H}}^{p}} & \leqslant 2^{1 / p}\left(\sum_{i=0}^{\infty}(i+1)^{p-1} \int_{0}^{G_{X}\left(\gamma_{i}\right) \wedge G_{X}\left(\gamma_{m+1}\right)} Q_{X}^{p}(u) d u\right)^{1 / p} \\
& \leqslant 2^{1 / p}\left(\sum_{i=0}^{\infty}(i+1)^{p-1} \int_{0}^{\gamma_{i} \wedge \gamma_{m+1}} Q_{X}^{p-1} \circ G_{X}(u) d u\right)^{1 / p},
\end{aligned}
$$

which implies that (6.11) (and hence $G(p))$ holds as soon as (3.4) does. 
Some examples showing that $G(p)$ and $D M(p, \gamma, X)$ cannot be compared. Consider the simple case $X_{n}=\sum_{i \geqslant 0} a_{i} \epsilon_{n-i}$, where $\left(\epsilon_{i}\right)_{i \in \mathbf{Z}}$ is a sequence of i.i.d. real-valued random variables with distribution $\mathscr{N}(0,1)$, and $\left(a_{i}\right)_{i \geqslant 0}$ is a sequence of real numbers such that $\sum_{i \geqslant 0} a_{i}^{2}<\infty$. Let $\mathscr{M}_{i}$ be the natural $\sigma$-algebra, that is, $\mathscr{M}_{i}=\sigma\left(\epsilon_{j}, j \leqslant i\right)$. Since $\mathbf{E}\left(X_{n} \mid \mathscr{M}_{0}\right)$ is Gaussian with mean 0 and variance $\sum_{i \geqslant n} a_{i}^{2}$, one has that

$$
\gamma_{n}=\sqrt{\frac{2}{\pi} \sum_{i \geqslant n} a_{i}^{2}}
$$

On the other hand, since $\left(\mathbf{E}\left(X_{n} \mid \mathscr{M}_{0}\right)\right)_{n \geqslant 0}$ is a Gaussian process, it follows that for any $p$ in $] 1,2[$, the condition $G(p)$ is equivalent to $G(2)$. Then, according to [12, condition (5.37)], we derive that for any $p$ in $] 1,2[$, the condition $G(p)$ holds if and only if

$$
\sum_{k=0}^{n} a_{k} \text { converges and } \sum_{i \geqslant 0}\left(\sum_{k=i}^{\infty} a_{k}\right)^{2}<\infty .
$$

Consequently, if $a_{i}=(-1)^{i}(\sqrt{i+1} \ln (i+1))^{-1}$, then the condition $G(p)$ holds for any $p$ in $] 1,2$ [, while $\sum_{i>0} \gamma_{i} / i=\infty$, so that $D M(p, \gamma, X)$ cannot holds for any $p$ in $] 1,2\left[\right.$. On the contrary, if $a_{i}=(i+1)^{-3 / 2}$, then $G(p)$ does not hold for any $p$ in ]1,2[, while $D M(p, \gamma, X)$ holds for any $p$ in ]1,2[ (to see this, apply item 2 of Corollary 1 with $r>1 /(2-p)$ ).

6.3. Proof of Proposition 1. We need the following preliminary result.

Proposition 4. Let $(\Omega, \mathscr{A}, \mathbf{P})$ be a probability space and let $\left(X_{k}\right)_{1 \leqslant k \leqslant n}$ be $n$ random variables with values in an $(r, D)$-smooth separable Banach space $\mathbf{B}$ such that $\mathbf{P}\left(\left\|X_{k}\right\|_{\mathbf{B}} \leqslant T\right)=1$ for any $1 \leqslant k \leqslant n$. For $i \leqslant n$, define the $\sigma$-algebras $\mathscr{M}_{i}$ by $\mathscr{M}_{i}=\{\varnothing, \Omega\}$ if $i \leqslant 0$ and $\mathscr{M}_{i}=\sigma\left(X_{j}, 1 \leqslant j \leqslant i\right)$ if $1 \leqslant i \leqslant n$. Let $S_{0}=0_{\mathrm{B}}$ and $S_{k}=\sum_{i=1}^{k}\left(X_{i}-\mathbf{E} X_{i}\right)$. Define the random variables $U_{i}$ by $U_{[n / q]+1}=S_{n}-S_{q[n / q]}$ and $U_{i}=S_{i q}-S_{i q-q}$ for $1 \leqslant i \leqslant[n / q]$. For any $x \geqslant T q$, the following inequality holds:

$$
\begin{aligned}
\mathbf{P}\left(\max _{1 \leqslant k \leqslant n}\left\|S_{k}\right\|_{\mathbf{B}} \geqslant 4 x\right) \leqslant & \frac{D}{x^{r}} \sum_{i=1}^{[n / q]+1} \mathbf{E}\left\|U_{i}-\mathbf{E}\left(U_{i} \mid \mathscr{M}_{(i-2) q}\right)\right\|_{\mathbf{B}}^{r} \\
& +\frac{1}{x} \sum_{i=3}^{[n / q]+1} \mathbf{E}\left\|\mathbf{E}\left(U_{i} \mid \mathscr{M}_{(i-2) q}\right)\right\|_{\mathbf{B}} .
\end{aligned}
$$

Proof of $\mathrm{Proposition} \mathrm{4.} \mathrm{Any} \mathrm{integer} j$ being distant at most $[q / 2]$ from an element of $q \mathbf{N}$, we have that

$$
\max _{1 \leqslant k \leqslant n}\left\|S_{k}\right\|_{\mathbf{B}} \leqslant 2\left[\frac{q}{2}\right] T+\max _{1 \leqslant j \leqslant[n / q]+1}\left\|\sum_{i=1}^{j} U_{i}\right\|_{\mathbf{B}} .
$$


Hence inequality (6.12) follows from the bound

$$
\begin{aligned}
\mathbf{P}\left(\max _{1 \leqslant j \leqslant[n / q]+1}\left\|\sum_{i=1}^{j} U_{i}\right\|_{\mathbf{B}} \geqslant 3 x\right) \leqslant & \frac{D}{x^{r}} \sum_{i=1}^{[n / q]+1} \mathbf{E}\left\|U_{i}-\mathbf{E}\left(U_{i} \mid \mathscr{M}_{(i-2) q}\right)\right\|_{\mathbf{B}}^{r} \\
& +\frac{1}{x} \sum_{i=3}^{[n / q]+1} \mathbf{E}\left\|\mathbf{E}\left(U_{i} \mid \mathscr{M}_{(i-2) q}\right)\right\|_{\mathbf{B}} .
\end{aligned}
$$

Consider the $\sigma$-algebras $\mathscr{F}_{i}^{U}=\mathscr{M}_{i q}$ and define the variables $\widetilde{U}_{i}$ as follows: $\widetilde{U}_{1}=U_{1}$, and $\widetilde{U}_{2 i-1}=U_{2 i-1}-\mathbf{E}\left(U_{2 i-1} \mid \mathscr{F}_{2(i-1)-1}^{U}\right)$ for $i>1, \widetilde{U}_{2}=U_{2}$ and $\widetilde{U}_{2 i}=U_{2 i}-\mathbf{E}\left(U_{2 i} \mid \mathscr{F}_{2(i-1)}^{U}\right)$ for $i>1$. Substituting $\widetilde{U}_{i}$ for $U_{i}$, we obtain the inequality

$$
\begin{aligned}
\max _{1 \leqslant j \leqslant[n / q]+1}\left\|\sum_{i=1}^{j} U_{i}\right\|_{\mathbf{B}} \leqslant & \max _{2 \leqslant 2 j \leqslant[n / q]+1}\left\|\sum_{i=1}^{j} \widetilde{U}_{2 i}\right\|_{\mathbf{B}}+\sum_{1 \leqslant 2 j-1 \leqslant[n / q]+1}\left\|\sum_{i=1}^{j} \widetilde{U}_{2 i-1}\right\|_{\mathbf{B}} \\
& +\sum_{i=1}^{[n / q]+1}\left\|U_{i}-\widetilde{U}_{i}\right\|_{\mathbf{B}}
\end{aligned}
$$

Note that $\left(\widetilde{U}_{2 i}\right)_{i \geqslant 1}$ (respectively, $\left.\left(\widetilde{U}_{2 i-1}\right)_{i \geqslant 1}\right)$ is a B-valued martingale difference sequence with respect to the filtration $\left(\mathscr{F}_{2 i}^{U}\right)_{i \geqslant 1}$ (respectively, $\left.\left(\mathscr{F}_{2 i-1}^{U}\right)_{i \geqslant 1}\right)$. Applying Doob's inequality to the submartingale $\left\|\sum_{i=1}^{j} \widetilde{U}_{2 i}\right\|_{\mathbf{B}}$ and next inequality (2.1), we obtain that

$$
\begin{aligned}
\mathbf{P}\left(\max _{2 \leqslant 2 j \leqslant[n / q]+1}\left\|\sum_{i=1}^{j} \widetilde{U}_{2 i}\right\|_{\mathbf{B}} \geqslant x\right) & \leqslant \frac{1}{x^{r}} \mathbf{E}\left\|\sum_{i=1}^{([n / q]+1) / 2} \widetilde{U}_{2 i}\right\|_{\mathbf{B}}^{r} \\
& \leqslant \frac{D}{x^{r}} \sum_{i=1}^{([n / q]+1) / 2} \mathbf{E}\left\|\widetilde{U}_{2 i}\right\|_{\mathbf{B}}^{r} .
\end{aligned}
$$

In the same way

$$
\mathbf{P}\left(\max _{1 \leqslant 2 j-1 \leqslant[n / q]+1}\left\|\sum_{i=1}^{j} \widetilde{U}_{2 i-1}\right\|_{\mathbf{B}} \geqslant x\right) \leqslant \frac{D}{x^{r}} \sum_{i=1}^{[n / q] / 2+1} \mathbf{E}\left\|\widetilde{U}_{2 i-1}\right\|_{\mathbf{B}}^{r} .
$$

On the other hand, we have

$$
\mathbf{P}\left(\sum_{i=1}^{[n / q]+1}\left\|U_{i}-\widetilde{U}_{i}\right\|_{\mathbf{B}} \geqslant x\right) \leqslant \frac{1}{x} \sum_{i=3}^{[n / q]+1} \mathbf{E}\left\|\mathbf{E}\left(U_{i} \mid \mathscr{M}_{(i-2) q}\right)\right\|_{\mathbf{B}} .
$$

Inequality (6.13) follows from (6.14)-(6.17). Proposition 4 is proved.

Now return to the proof of Proposition 1. For every $v \in[0,1]$, we introduce the variables

$$
X_{i}^{\prime}=X_{i} \mathbb{I}\left(\left\|X_{i}\right\|_{\mathbf{H}} \leqslant Q(v)\right) \quad \text { and } \quad X_{i}^{\prime \prime}=X_{i} \mathbb{I}\left(\left\|X_{i}\right\|_{\mathbf{H}}>Q(v)\right) .
$$


Let $S_{n}^{\prime}=\sum_{i=1}^{n}\left(X_{i}^{\prime}-\mathbf{E} X_{i}^{\prime}\right)$ and $S_{n}^{\prime \prime}=\sum_{i=1}^{n}\left(X_{i}^{\prime \prime}-\mathbf{E} X_{i}^{\prime \prime}\right)$ and write

$$
\begin{aligned}
\mathbf{P}\left(\max _{1 \leqslant k \leqslant n}\left\|S_{k}\right\|_{\mathbf{H}} \geqslant 5 x\right) \leqslant & \mathbf{P}\left(\max _{1 \leqslant k \leqslant n}\left\|S_{k}^{\prime}\right\|_{\mathbf{H}} \geqslant 4 x\right) \\
& +\mathbf{P}\left(\max _{1 \leqslant k \leqslant n}\left\|S_{k}^{\prime \prime}\right\|_{\mathbf{H}} \geqslant x\right) .
\end{aligned}
$$

Arguing as for (6.4) and using the fact that

$$
u<L(Q(v)) \Longleftrightarrow Q(v)<Q(u) \Longleftrightarrow u<v,
$$

we obtain that

$$
\mathbf{P}\left(\max _{1 \leqslant k \leqslant n}\left\|S_{k}^{\prime \prime}\right\|_{\mathbf{H}} \geqslant x\right) \leqslant \frac{2 n}{x} \int_{0}^{v} Q(u) d u .
$$

To control the first term in decomposition (6.18), we apply inequality (6.12) with $r=2$ and $D=1$. Since in Hilbert spaces, $\mathbf{E}\left\|U_{i}^{\prime}-\mathbf{E}\left(U_{i}^{\prime} \mid \mathscr{M}_{(i-2) q}\right)\right\|_{\mathbf{H}}^{2} \leqslant$ $\mathbf{E}\left\|U_{i}^{\prime}\right\|_{\mathbf{H}}^{2}$, we get that for any integer $q$ smaller than $n$ and any $x \geqslant q Q(v)$,

$\mathbf{P}\left(\max _{1 \leqslant k \leqslant n}\left\|S_{k}^{\prime}\right\|_{\mathbf{H}} \geqslant 4 x\right) \leqslant \frac{1}{x^{2}} \sum_{i=1}^{[n / q]+1} \mathbf{E}\left\|U_{i}^{\prime}\right\|_{\mathbf{H}}^{2}+\frac{1}{x} \sum_{i=3}^{[n / q]+1} \mathbf{E}\left\|\mathbf{E}\left(U_{i}^{\prime} \mid \mathscr{M}_{(i-2) q}\right)\right\|_{\mathbf{H}}$,

where $U_{i}^{\prime}=S_{i q}^{\prime}-S_{i q-q}^{\prime}$ for $1 \leqslant i \leqslant[n / q]$, and $U_{[n / q]+1}^{\prime}=S_{n}^{\prime}-S_{q[n / q]}^{\prime}$. Define the random variables

$$
Y_{k}=X_{k}-\mathbf{E} X_{k}, \quad Y_{k}^{\prime}=X_{k}^{\prime}-\mathbf{E} X_{k}^{\prime}, \quad \text { and } \quad Y_{k}^{\prime \prime}=X_{k}^{\prime \prime}-\mathbf{E} X_{k}^{\prime \prime} .
$$

For all $1 \leqslant i \leqslant[n / q]$, we have that

$$
\begin{array}{rl}
\mathbf{E}\left\|U_{i}^{\prime}\right\|_{\mathbf{H}}^{2} & =\sum_{j=(i-1)}^{i q} \sum_{q+1}^{i q} \mathbf{E} \mathbf{\ell}\langle(i-1) q+1 \\
& \left.=\sum_{j=(i-1)}^{i q} \sum_{\ell}^{\prime}, Y_{j}^{\prime}\right\rangle_{\mathbf{H}} \\
\sum_{\ell=(i-1)}^{i q} q+1 & \mathbf{E}\left\langle X_{\ell}^{\prime}, Y_{j}^{\prime}\right\rangle_{\mathbf{H}} .
\end{array}
$$

Let $1 \leqslant i \leqslant[n / q]$. Clearly

$$
\mathbf{E}\left\|U_{i}^{\prime}\right\|_{\mathbf{H}}^{2} \leqslant 2 \sum_{j=(i-1)}^{i q} \sum_{q+1}^{j} \mathbf{E}\left(\left\|X_{\ell}^{\prime}\right\|_{\mathbf{H}}\left\|\mathbf{E}\left(Y_{j}^{\prime} \mid \mathscr{M}_{\ell}\right)\right\|_{\mathbf{H}}\right) \leqslant A_{1}+A_{2},
$$

where

$$
\begin{aligned}
& A_{1}=\sum_{j=(i-1)}^{i q} \sum_{q+1}^{j} 2 \mathbf{E}\left(\left\|X_{\ell}^{\prime}\right\|_{\mathbf{H}}\left\|\mathbf{E}\left(Y_{j} \mid \mathscr{M}_{\ell}\right)\right\|_{\mathbf{H}}\right), \\
& A_{2}=\sum_{j=(i-1){ }_{i-1)} \sum_{q+1}}^{j q} \sum_{\ell=(i-1)}^{j} 2 \mathbf{E}\left(\left\|X_{\ell}^{\prime}\right\|_{\mathbf{H}}\left\|\mathbf{E}\left(Y_{j}^{\prime \prime} \mid \mathscr{M}_{\ell}\right)\right\|_{\mathbf{H}}\right) .
\end{aligned}
$$


Since $\left\|X_{k}^{\prime}\right\|_{\mathbf{H}} \leqslant Q(v)$, it follows that

$$
\begin{aligned}
\frac{A_{2}}{2 Q(v)} & \leqslant \sum_{j=(i-1)}^{i q} \sum_{\ell+1}^{j} \mathbf{E}\left\|\mathbf{E}\left(Y_{j}^{\prime \prime} \mid \mathscr{M}_{\ell}\right)\right\|_{\mathbf{H}} \\
& \leqslant \sum_{j=(i-1) q+1}^{i q}(j-(i-1) q) \mathbf{E}\left\|Y_{j}^{\prime \prime}\right\|_{\mathbf{H}} \\
& \leqslant 2 \sum_{j=(i-1) q+1}^{i q}(j-(i-1) q) \mathbf{E}\left\|X_{j}^{\prime \prime}\right\|_{\mathbf{H}} .
\end{aligned}
$$

Next arguing as in (6.3) and using (3.13), we easily obtain that for all $j \geqslant 1$,

$$
\mathbf{E}\left\|X_{j}^{\prime \prime}\right\|_{\mathbf{H}} \leqslant \int_{0}^{v} Q(u) d u .
$$

It follows that

$$
A_{2} \leqslant 2 q(q+1) Q(v) \int_{0}^{v} Q(u) d u .
$$

On the other hand, applying [8, inequality (3.29)], we infer that

$$
A_{1} \leqslant 2 \sum_{j=(i-1) q+1}^{i q} \sum_{\ell=(i-1) q+1}^{j} \int_{0}^{\gamma_{j-\ell}} Q_{\left\|X_{\ell}^{\prime}\right\|_{\mathbf{H}}} \circ G_{\left\|Y_{j}\right\|_{\mathbf{H}}}(u) d u .
$$

Note that, for all $j \geqslant 1, Q_{\left\|Y_{j}\right\|_{\mathbf{H}}} \leqslant Q_{\left\|X_{j}\right\|_{\mathbf{H}}}+\mathbf{E}\left\|X_{j}\right\|_{\mathbf{H}}$. Arguing as in (4.4), we infer that $G_{\left\|Y_{j}\right\|_{\mathbf{H}}}(u) \geqslant G(u / 2)$. From this inequality and (6.24) we obtain the bound

$$
A_{1} \leqslant 4 \sum_{j=(i-1)}^{i q} \sum_{q+1}^{j} \int_{\ell=(i-1) q+1}^{\left(\gamma_{j-\ell}\right) / 2} Q_{\left\|X_{\ell}^{\prime}\right\|_{\mathrm{H}}} \circ G(u) d u .
$$

Since $H$ is absolutely continuous and monotonic, we can make the changeof-variables $v=H(u)$ (see [24, Theorem 6.26] and the example given on page 156 therein), which yields

$$
A_{1} \leqslant 4 \sum_{j=(i-1)}^{i q} \sum_{q+1}^{j} \int_{\ell=(i-1) q+1}^{G\left(\gamma_{j-\ell} / 2\right)} Q_{\left\|X_{\ell}^{\prime}\right\|_{\mathbf{H}}}(u) Q(u) d u .
$$

Applying (6.7), we infer that for any $\ell \geqslant 1$ and any $u \in[0,1]$,

$$
Q_{\left\|X_{\ell}^{\prime}\right\|_{\mathbf{H}}}(u) \leqslant Q(v \vee u) .
$$

Using (6.25), we get that

$$
A_{1} \leqslant 4 \sum_{k=0}^{q-1}(q-k) \int_{0}^{G\left(\gamma_{k} / 2\right)} Q(v \vee u) Q(u) d u .
$$


Starting from (6.21) and collecting (6.23) and (6.26), we infer that for all $1 \leqslant i \leqslant[n / q]$,

$$
\begin{aligned}
\mathbf{E}\left\|U_{i}^{\prime}\right\|_{\mathbf{H}}^{2} \leqslant 4 q( & 2 q Q(v) \int_{0}^{v} Q(u) d u \\
& \left.+\int_{0}^{1}\left(\left(\frac{\gamma}{2}\right)^{-1} \circ G^{-1}\right)(u) Q^{2}(u) \mathbb{I}(v \leqslant u \leqslant 1) d u\right) .
\end{aligned}
$$

On the other hand, for all $3 \leqslant i \leqslant[n / q]$,

$$
\mathbf{E}\left\|\mathbf{E}\left(U_{i}^{\prime} \mid \mathscr{M}_{(i-2) q}\right)\right\|_{\mathbf{H}} \leqslant \mathbf{E}\left\|\mathbf{E}\left(U_{i} \mid \mathscr{M}_{(i-2) q}\right)\right\|_{\mathbf{H}}+2 \sum_{k=(i-1) q+1}^{i q} \mathbf{E}\left\|X_{k}^{\prime \prime}\right\|_{\mathbf{H}}
$$

Applying inequality (6.22), we obtain that

$$
\sum_{k=(i-1) q+1}^{i q} \mathbf{E}\left\|X_{k}^{\prime \prime}\right\|_{\mathbf{H}} \leqslant q \int_{0}^{v} Q(u) d u
$$

According to the definition of the coefficients $\gamma_{i}$, we have that

$$
\mathbf{E}\left\|\mathbf{E}\left(U_{i} \mid \mathscr{M}_{(i-2) q}\right)\right\|_{\mathbf{H}} \leqslant q \gamma_{q}=2 q H \circ G\left(\frac{\gamma_{q}}{2}\right)=2 q \int_{0}^{G\left(\gamma_{q} / 2\right)} Q(u) d u .
$$

Starting from (6.28), we infer that for all $3 \leqslant i \leqslant[n / q]$,

$$
\mathbf{E}\left\|\mathbf{E}\left(U_{i}^{\prime} \mid \mathscr{M}_{(i-2) q}\right)\right\|_{\mathbf{H}} \leqslant 2 q\left(\int_{0}^{G\left(\gamma_{q} / 2\right)} Q(u) d u+\int_{0}^{v} Q(u) d u\right) .
$$

The terms involving the quantity $U_{[n / q]+1}^{\prime}$ are treated similarly: we obtain the same bound as (6.27) and (6.29) but with $n-q[n / q]$ instead of $q$. Starting from inequality (6.18) and collecting (6.19), (6.20), (6.27), and (6.29), we obtain that for any $v \in[0,1]$, any positive integer $q$ smaller than $n$, and any $x \geqslant q Q(v)$,

$$
\begin{aligned}
\mathbf{P}\left(\max _{1 \leqslant k \leqslant n}\left\|S_{k}\right\|_{\mathbf{H}} \geqslant 5 x\right) \leqslant & \frac{12 n}{x} \int_{0}^{v} Q(u) d u+\frac{2 n}{x} \int_{0}^{G\left(\gamma_{q} / 2\right)} Q(u) d u \\
& +\frac{4 n}{x^{2}} \int_{v}^{1}\left(\left(\frac{\gamma}{2}\right)^{-1} \circ G^{-1}\right)(u) Q^{2}(u) d u
\end{aligned}
$$

Now choose $v=S(x / r)$ and $q=\left((\gamma / 2)^{-1} \circ G^{-1}(v)\right) \wedge n$. This choice implies that $G\left(\gamma_{q} / 2\right) \leqslant v$ and that

$$
q Q(v)=R(v)=R\left(S\left(\frac{x}{r}\right)\right) \leqslant \frac{x}{r} \leqslant x .
$$

Applying inequality (6.30), we obtain the desired result. 
6.4. Proof of Lemma 1. Take $f \in W_{1}(\mu)$. We first check that under (4.1), $|f|$ is integrable with respect to $d F$. Without loss of generality assume that $f(0)=0$. Clearly

$$
\int|f| d F \leqslant \int_{\mathbf{R}^{+}}\left(\int_{[0, t]}|g(x)| \mu(d x)\right) d F(t)+\int_{\mathbf{R}^{-}}\left(\int_{[t, 0[}|g(x)| \mu(d x)\right) d F(t) .
$$

Applying Fubini's theorem, we obtain that

$$
\int|f| d F \leqslant \int_{\mathbf{R}^{+}}|g(x)|(1-F(x)) \mu(d x)+\int_{\mathbf{R}^{-}}|g(x)| F(x) \mu(d x) .
$$

Since $g$ belongs to $\mathbf{L}^{2}(\mu)$, the right-hand side is finite as soon as (4.1) holds. In the same way, we have both

and

$$
\int f d F=\int_{\mathbf{R}^{+}} g(x)(1-F(x)) \mu(d x)-\int_{\mathbf{R}^{-}} g(x) F(x) \mu(d x)
$$

$$
\int f d G=\int_{\mathbf{R}^{+}} g(x)(1-G(x)) \mu(d x)-\int_{\mathbf{R}^{-}} g(x) G(x) \mu(d x) .
$$

Consequently,

$$
\int f d F-\int f d G=\int g(x)(G(x)-F(x)) \mu(d x) .
$$

The result follows by noting that

$$
D(F, G, \mu)=\sup _{\|g\|_{\mathbf{L}^{2}(\mu)} \leqslant 1}\left|\int_{\mathbf{R}} g(x)(F(x)-G(x)) d \mu(x)\right| .
$$

Acknowledgment. The authors are indebted to the referee for careful reading the manuscript and for helpful comments.

\section{REFERENCES}

1. de Acosta $A$. Inequalities for $\mathrm{B}$-valued random vectors with applications to the strong law of large numbers. - Ann. Probab., 1981, v. 9, № 1, p. 157-161.

2. Assouad $P$. Espaces $p$-lisses et $q$-convexes, inégalités de Burkholder. - Séminaire Maurey-Schwartz 1974-1975: Espaces $L^{p}$, applications radonifiantes et géométrie des espaces de Banach, Exp. №XV. Paris: Centre Math., École Polytech., 1975, 8 p.

3. Baum L. E., Katz M. Convergence rates in the law of large numbers. - Trans. Amer. Math. Soc., 1965 , v. 120 , p. 108-123.

4. Berbee H. C. P. Convergence rates in the strong law for bounded mixing sequences. Probab. Theory Related Fields, 1987, v. 74, № 2, p. 255-270.

5. Bosq D. Linear Processes in Function Spaces. Theory and Applications. New York: Springer-Verlag, 2000, 283 p. (Lecture Notes in Statist., v. 149.)

6. Bradley R.C. Introduction to strong mixing conditions, v. 1. Technical Report. Bloomington: Dept. of Math., Indiana Univ., Custom Publishing of I. U., 2002.

7. Dedecker J., Doukhan P. A new covariance inequality and applications. - Stochastic Process. Appl., 2003, v. 106, № 1, p. 63-80.

8. Dedecker J., Merlevède $F$. The conditional central limit theorem in Hilbert spaces. Stochastic Process. Appl., 2003, v. 108, № 2, p. 229-262. 
9. Dedecker J., Prieur C. New dependence coefficients. Examples and applications to statistics. - Probab. Theory Related Fields, 2005, v. 132, № 2, p. 203-236.

10. Elton $J$. A law of large numbers for identically distributed martingale differences. Ann. Probab., 1981, v. 9, № 3, p. 405-412.

11. Гордин М. И. О центральной предельной теореме для стационарных процессов. Докл. АН ССCР, 1969, т. 188, № 4, с. 739-741.

12. Hall P., Heyde C. C. Martingale Limit Theory and its Applications. New YorkLondon: Academic Press, 1980, 308 p.

13. Hipp C. Convergence rates of the strong law for stationary mixing sequences. Z. Wahrscheinlichkeitstheor. Verw. Geb. 1979, v. 49, № 1, p. 49-62.

14. Lai T. L. Convergence rates and $r$-quick versions of the strong law for stationary mixing sequences. - Ann. Probab., 1977, v. 5, p. 693-706.

15. Lesigne E., Volny D. Large deviations for martingales. - Stochastic Process. Appl., 2001 , v. 96 , № 1 , p. 143-159.

16. McLeish D. L. A maximal inequality and dependent strong laws. - Ann. Probab., 1975 , v. 3, № 5 , p. 829-839.

17. Merlevède $F$. On the central limit theorem and its weak invariance principle for strongly mixing sequences with values in a Hilbert space via martingale approximation. - J. Theoret. Probab., 2003, v. 16, № 3, p. 625-653.

18. Mourier E. Éléments aléatoires dans un espace de Banach. - Ann. Inst. H. Poincaré, 1953, v. 13, p. 161-244.

19. Peligrad $M$. Convergence rates of the strong law for stationary mixing sequences. Z. Wahrscheinlichkeitstheor. Verw. Geb., 1985, v. 70, № 2, p. 307-314.

20. Peligrad $M$. The $r$-quick version of the strong law for stationary $\varphi$-mixing sequences. - Almost Everywhere Convergence (Columbus, 1988). Boston: Academic Press, 1989, p. 335-348.

21. Pisier $G$. Martingales with values in uniformly convex spaces. - Israel J. Math., 1975 , v. 20, № 3-4, p. 326-350.

22. Rio E. A maximal inequality and dependent Marcinkiewicz-Zygmund strong laws. Ann. Probab., 2002, v. 23, № 2, p. 918-937.

23. Rio $E$. Théorie asymptotique des processus aléatoires faiblement dépendants. Berlin: Springer-Verlag, 2000, 169 p. (Math. Appl. (Berlin), v. 31.)

24. Rosenblatt $M$. A central limit theorem and a strong mixing condition. - Proc. Natl. Acad. Sci. USA, 1956, v. 42, p. 43-47.

25. Rudin W. Real and Complex Analysis. New York: McGraw-Hill, 1987, 416 p.

26. Shao Q.-M. Complete convergence for $\alpha$-mixing sequences. - Statist. Probab. Lett., 1993, v. 16, № 4, p. 279-287.

27. Woyczyński W. A. A central limit theorem for martingales in Banach spaces. - Bull. Acad. Polon. Sci., 1975, v. 23, № 8, p. 917-920.

28. Woyczyński W. A. Laws of large numbers for vector-valued martingales. - Bull. Acad. Polon. Sci., 1975, v. 23, № 11, p. 1199-1201.

29. Woyczyński W. A. Asymptotic behavior of martingales in Banach spaces. II. - Martingale Theory in Harmonic Analysis and Banach Spaces (Cleveland, 1981). BerlinNew York: Springer, 1982, p. 216-225. (Lecture Notes in Math., v. 939.)

Поступила в редакцию 13.XI.2003

Исправленный вариант

13.III.2006 